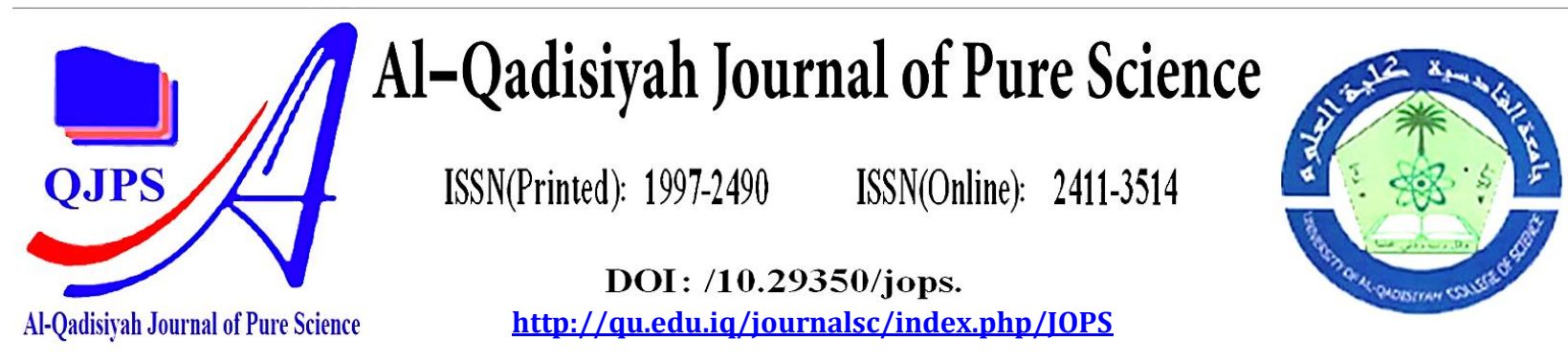

\title{
Influence of Temperature and Concentration on MHD Oscillatory Flow for Bingham Fluid with Variable Viscosity Through an inclined channel
}

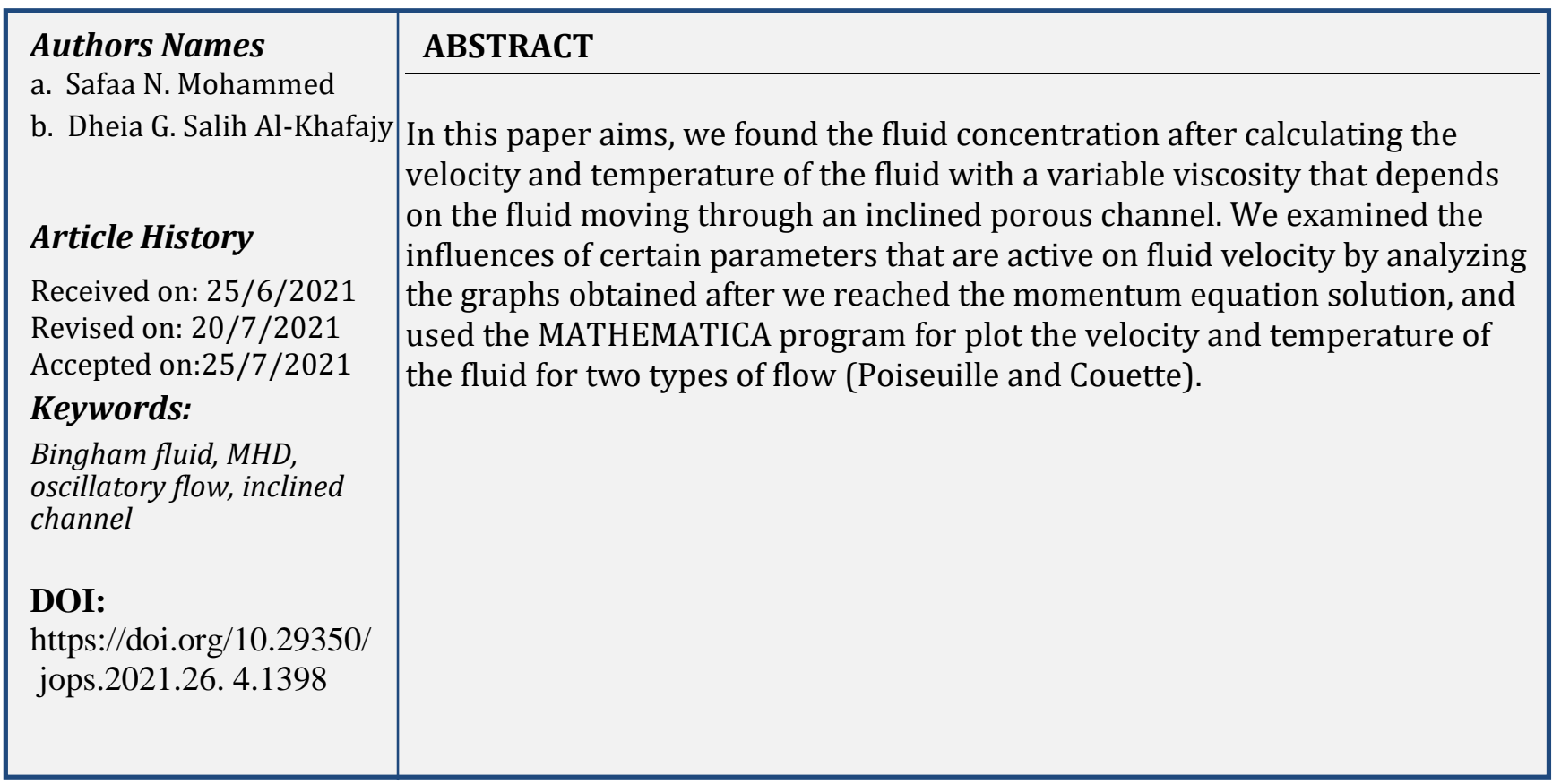

\section{Introduction}

The study of the flow of an electrically conductive fluid has many applications in engineering problems such as (MHD) generators, Plasma studies, nuclear reactors, geothermal energy extraction and boundaries class control in the field of aerodynamics [1]. Viscosity is one of the most important specifications in fluids. The viscosity of oil derivatives is an important physical property in many of such derivatives, especially oils and lubricants. Additionally, the viscosity of the crude oil and its derivatives is technically important and plays a prominent role in the flow calculation. Flow through a porous medium, under the influence of temperature variations, is one of the most important contemporary topics because it finds great applications in geophysics and

\footnotetext{
a Department of Mathematics, College of Science, University of Al-Qadisiyah, Diwaniyah, Iraq, E-Mail: andjdkfrwed123456789@gmail.com

b Department of Mathematics, College of Science, University of Al-Qadisiyah, Diwaniyah, Iraq, E-Mail: dr.dheia.g.salih@gmail.com , dheia.salih@qu.edu.iq
} 
technology. The study of the flow of sedimentary liquids is of practical importance, especially flow through packed beds, sedimentation, environmental pollution, and central filtering of particles. There are many studies in the scientific literature on fluid movement in the channel, for examples; [2] study the effect of heat transfer on the oscillating flow of the hydrodynamics magnetizing Eyring-Powell fluid through a porous medium. In [3] examined the influence of heat transfer on (MHD) for the oscillatory flow of Williamson fluid with variable viscosity model for two kinds of geometries "Poiseuille flow and Couette flow" through a porous medium channel. By [4] an investigation of peristaltic flow of Bingham plastic fluid in an inclined tapered asymmetric channel with variable viscosity. The effect of heat transfer on the (MHD) oscillatory flow of a Jeffrey fluid with variable viscosity model through porous medium studied by Al-Khafajy [5]. Adnan and Abdulhadi [6] analyzed the effect of an inclined magnetic field on peristaltic flow of Bingham plastic fluid in an inclined symmetric channel with slip conditions. Ara et al. [7] explored the Jeffery- Hamel flow of Bingham plastic fluid in converging channel in the presence of external magnetic field. However, Salih [8] illustrated the influence of varying temperature and concentration on (MHD) peristaltic transport of Jeffery fluid with variable viscosity through porous channel. It is naturally found in human living body such as urine movement from kidney to bladder, food swallowing process and blood flow in the small vessels [9], [10] and [11]. The study considers a mathematical model for the influence of MHD oscillatory slip flow for Bingham fluid with variable viscosity through an inclined channel with varying temperature and concentration. The perturbation technique series use to solve the problem. The result of the physical parameters problem was discussed by using the graphs.

\section{Mathematical Formulation}

The flow of a Bingham fluid in a channel of width $\mathrm{h}$ is assumed by the researcher. Flow the fluid under the effects of electrically applied magnetic field and radioactive heat transfer as in (Fig 1). Assume that the fluid has small produced electromagnetic force and the electrical conductivity is small. Cartesian coordinate system can be considered such that $(u(y), 0,0)$ is a velocity vector in which $u$ is the $\mathrm{x}$-component of velocity and $y$ is perpendicular to the $\mathrm{x}$-axis. Fluid flow under one condition, no-slip condition, will be studied by the researcher.

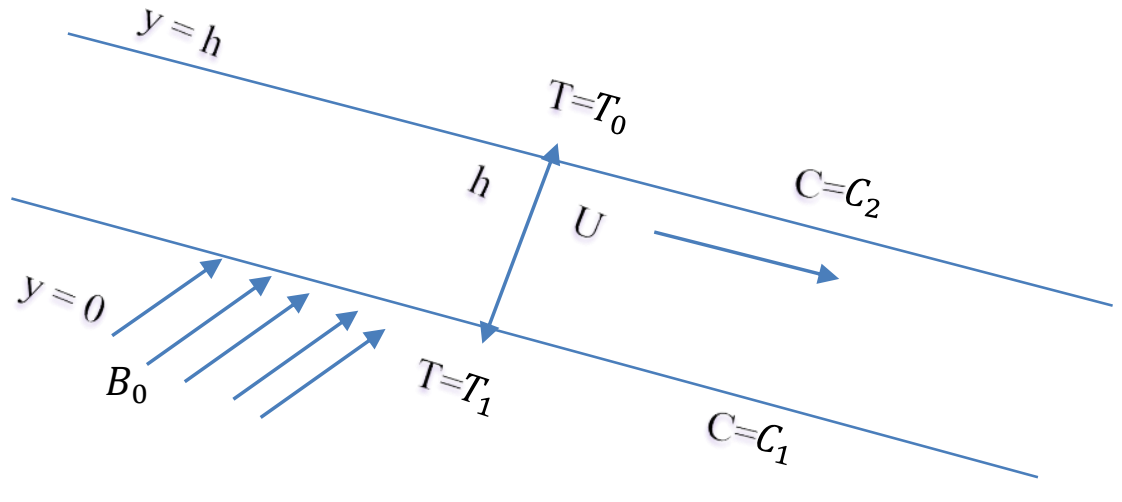

Figure 1 Physical model

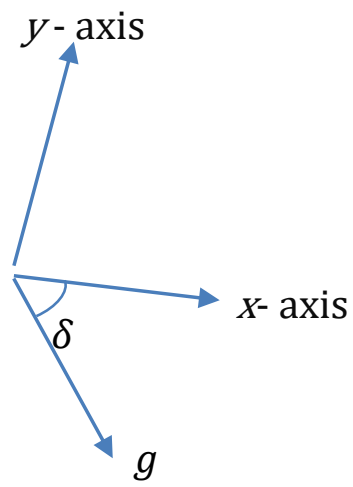


The essential equations governing the problem are provided as:

The continuity equation is given by: $\frac{\partial \bar{u}}{\partial \bar{x}}+\frac{\partial \bar{v}}{\partial \bar{y}}=0$

The momentum equations are:

In the $\mathrm{x}$ - direction: $\rho\left(\frac{\partial \overline{\mathrm{u}}}{\partial \overline{\mathrm{t}}}+\overline{\mathrm{u}} \frac{\partial \overline{\mathrm{u}}}{\partial \overline{\mathrm{x}}}+\overline{\mathrm{v}} \frac{\partial \overline{\mathrm{u}}}{\partial \overline{\mathrm{y}}}\right)=-\frac{\partial \overline{\mathrm{p}}}{\partial \overline{\mathrm{x}}}+\frac{\partial \overline{\mathcal{S}}_{\overline{\mathrm{xy}}}}{\partial \overline{\mathrm{y}}}-\sigma \mathrm{B}_{0}^{2} \operatorname{Sin}^{2}(\xi) \overline{\mathrm{u}}-\frac{\mu(\overline{\mathrm{y}})}{\mathrm{k}} \overline{\mathrm{u}}+\rho \sin (\delta)$,

In the $y$-direction: $\rho\left(\frac{\partial \bar{v}}{\partial \bar{t}}+\bar{u} \frac{\partial \bar{v}}{\partial \bar{x}}+\bar{v} \frac{\partial \bar{v}}{\partial \bar{y}}\right)=-\frac{\partial \bar{p}}{\partial \bar{y}}+\frac{\partial \bar{\delta}_{\bar{x} \bar{y}}}{\partial \bar{x}}-\sigma B_{0}^{2} \operatorname{Sin}^{2}(\xi) \bar{v}-\frac{\mu(\bar{y})}{k} \bar{v}+\rho g \cos (\delta)$,

The temperature equation is given by:

$c_{T} \rho\left(\frac{\partial T}{\partial \bar{t}}+\bar{u} \frac{\partial T}{\partial \bar{x}}+\bar{v} \frac{\partial T}{\partial \bar{y}}\right)=K_{T}\left(\frac{\partial^{2} T}{\partial \bar{x}^{2}}+\frac{\partial^{2} T}{\partial \bar{y}^{2}}\right)+\overline{\mathcal{S}} \cdot(\operatorname{grad} \bar{V})-\frac{\partial q}{\partial y}$

The concentration equation:

$\frac{\partial C}{\partial \bar{t}}=D \frac{\partial^{2} C}{\partial \bar{y}^{2}}-K_{r}^{*}\left(C-C_{2}\right)+\frac{D K_{T}}{T_{m}} \frac{\partial^{2} T}{\partial \bar{y}^{2}}$.

where $V \equiv(u(y, t), 0,0)$ is "the velocity field", $T(y, t)$ is "a fluid temperature", $\mu_{(\bar{y})}$ is "a fluid viscosity dependent on the traveled distance", $B_{0}$ is "a magnetic field strength", $\mu_{c}$ the "magnetic permeability", $\rho$ is "a fluid density", $\mu_{p}$ the "magnetic permeability", $\sigma$ is "a conductivity of the fluid", $k$ is "a permeability", $c_{T}$ is "a specific heat at constant pressure", $D$ is "the coefficient of mass diffusivity", $K_{T}$ is "a thermal conductivity" and $q$ is "a radioactive heat flux".

The temperatures and concentration at the walls of the channel are given as:

$T=T_{0}, C=C_{2}$ at $\bar{y}=0$ and $T=T_{1}, C=C_{1}$ at $\bar{y}=h$

The radioactive heat flux [6] is given by:

$\frac{\partial q}{\partial y}=4 \eta^{2}\left(T_{0}-T\right)$

here $\eta$ denotes the radiation absorption.

The basic equation for the Bingham fluid is given as:

$\overline{\boldsymbol{S}}=\left\{\begin{array}{ccc}\mu(\bar{y}) \bar{X}+\frac{\tau_{0}}{\bar{\gamma}} \bar{X} & \text { for } & \tau \geq \tau_{0} \\ 0 & \text { for } & \tau<\tau_{0}\end{array}\right.$

Where $\mu_{(\bar{y})}$ is a fluid viscosity dependent on the traveled distance, $\tau_{0}$ is the yield stress, and $\dot{\gamma}$ is defined as:

$\dot{\gamma}=\sqrt{\frac{1}{2} \sum_{i} \sum_{j} \dot{\gamma}_{i j} \dot{\gamma}_{j i}}=\sqrt{\frac{1}{2} \Pi}$

here $\prod$ is the second invariant strain tensor.

The Rivlin-Ericksen tensors are given as $\bar{X}=\nabla \bar{V}+(\nabla \bar{V})^{T}$, where $(\nabla \overline{\mathrm{V}})$ is the velocity field in the cartesian coordinates system $(x, y, z)$ and $(\nabla \bar{V})^{\mathrm{T}}$ is the transpose of the velocity field. 
The stress component are given by:

$\overline{\boldsymbol{S}}_{\overline{x x}}=\overline{\boldsymbol{S}}_{\overline{y y}}=0, \overline{\boldsymbol{S}}_{\overline{x y}}=\overline{\boldsymbol{S}}_{\overline{y x}}=\mu(\bar{y}) \bar{u}_{y}+\tau_{0}$

and $\overline{\mathcal{S}} \cdot(\operatorname{grad} \bar{V})=\overline{\mathcal{S}}_{x y} \bar{u}_{\bar{y}}$

So that the heat equation become

$c_{T} \rho \frac{\partial T}{\partial \bar{t}}=K_{T} \frac{\partial^{2} T}{\partial \bar{y}^{2}}+\overline{\mathcal{S}}_{x y} \bar{u}_{\bar{y}}-4 \eta^{2}\left(T_{0}-T\right)$

\section{Method of Solution}

The governing equations of the motion, we may introduce the non-dimensional conditions are as follows:

$$
\left.\begin{array}{c}
K_{r}=\frac{h K_{r}^{*}}{U}, S_{r}=\frac{D K_{T}\left(T_{1}-T_{0}\right)}{U T_{m} h\left(C_{1}-C_{2}\right)}, S_{c}=\frac{U h}{D}, F r=\frac{U^{2}}{g h}, \Phi=\frac{C-C_{2}}{C_{1}-C_{2}}, \\
x=\frac{\bar{x}}{h}, y=\frac{\bar{y}}{h}, u=\frac{\bar{u}}{U}, t=\frac{\bar{t} U}{h}, p=\frac{\bar{p} h}{\mu_{c} U}, M^{2}=\frac{\sigma B_{0}^{2} h^{2}}{\mu_{c}}, D a=\frac{k}{h^{2}}, \dot{\gamma}=\frac{h}{U} \overline{\dot{\gamma}}, \\
\Delta T=T_{1}-T_{0}, \theta=\frac{T-T_{0}}{\Delta T}, B n=\frac{h \tau_{0}}{\mu_{c} U}, \mathcal{S}=\frac{h}{\mu_{c} U} \overline{\mathcal{S}}, \operatorname{Pr}=\frac{\mu_{c} c_{p}}{K_{T}}, E c=\frac{U^{2}}{c_{p} \Delta T}, \\
\mu(y)=\frac{\mu(\bar{y})}{\mu_{c}}, B r=\operatorname{Pr} E c, \operatorname{Re}=\frac{\rho h U}{\mu_{c}}, P e=\frac{\rho h U c_{p}}{K_{T}}, N^{2}=\frac{4 \eta^{2} h^{2}}{K_{T}}
\end{array}\right\}
$$

where $(U)$ is "the mean flow velocity", $(D a)$ is "Darcy number", $(R e)$ is "Reynolds number", $(M)$ is "magnetic parameter", $(\mathrm{Pe})$ is "the Peclet number" and $(N)$ is" the radiation parameter", (Bn) "Bingham number", (Br) "Brinkman number", (Pr) "Prandtl number", (Ec) "Eckert number", $\mu(y)$ is "the fluid viscosity dependent on the traveled distance", $\left(K_{r}\right)$ "Chemical reaction parameter", $\left(S_{r}\right)$ "Soret number", $\left(S_{c}\right)$ "Schmidt number", $(F r)$ "Froude number".

Substituting equations (12) into equations (1)-(6), (9), and (11), we have the following of nondimensional equations:

$\frac{\partial u}{\partial x}+\frac{\partial v}{\partial y}=0$

$\frac{\partial p}{\partial x}=-R e \frac{\partial u}{\partial t}+\frac{\partial}{\partial y}\left(\mu(y) \frac{\partial u}{\partial y}+B n\right)-\left(M_{1}^{2}+\frac{\mu(y)}{D a}\right) u+\frac{R e}{F r} \sin (\delta)$

$P e \frac{\partial \theta}{\partial t}=\frac{\partial^{2} \theta}{\partial y^{2}}+N^{2} \theta+(\mu(y)) B r\left(\frac{\partial u}{\partial y}\right)^{2}+B r B n \frac{\partial u}{\partial y}$

$\frac{\partial \Phi}{\partial t}=\frac{1}{S_{c}} \frac{\partial^{2} \Phi}{\partial y^{2}}-K_{r} \Phi+S r \frac{\partial^{2} \theta}{\partial y^{2}}$

and $\mathcal{S}_{x y}=\mu(y) \frac{\partial u}{\partial y}+\mathrm{Bn}$

\section{Reynold's Model of Viscosity}

The Reynold's model and variation of viscosity with dependent on the traveled distance are defined as: 
$\mu(y)=e^{-\alpha y}$

By using the Maclaurin series, we get:

$\mu(y)=1-\alpha y, \quad \alpha<<1$

In this case, the viscosity is fixed at $\alpha=0$, by substituting equation (18) by equation (14), we get:

$\frac{\partial p}{\partial x}=-R e \frac{\partial u}{\partial t}+\left((1-\alpha y) \frac{\partial^{2} u}{\partial y^{2}}-\alpha \frac{\partial u}{\partial y}\right)-\left(M_{1}^{2}+\frac{1-\alpha y}{D a}\right) u+\frac{R e}{F r} \sin (\delta)$

where $M_{1}=\operatorname{MSin}(\xi)$.

\section{Solution of the Problem}

This section contains a solution of the heat equation and motion equation and concentration equation.

\subsection{Solution of motion equation}

\subsubsection{Poiseuille flow}

We will find a solution to the equation of motion (14) for two types of flows "Poiseuille and Couette". First let

$u(y, t)=u_{0}(y) e^{i \omega t},-\frac{\partial p}{\partial x}=\lambda e^{i \omega t}$

where $\omega$ is a frequency of the oscillation and $\lambda$ is a real constant.

Substituting equations (20) into equation (14), we have

$\operatorname{Re} i \omega u_{0}(y)=\lambda+\left((1-\alpha y) \frac{\partial^{2}}{\partial y^{2}} u_{0}(y)-\alpha \frac{\partial}{\partial y} u_{0}(y)\right)-\left(M_{1}{ }^{2} \frac{(1-\alpha y)}{D a}\right) u_{0}(y)+\frac{R e}{F r} \sin (\delta) e^{-i \omega t}$

Equation (20) is a linear differential equation and it is hard to find an exact solution, so will use the perturbation technique to find the solution of problem as follows:

$$
u_{0}(y)=u_{00}(y)+\alpha u_{01}(y)
$$

Now, by substituting equation (22) in equation (21), we obtain:

$\frac{\partial^{2} u_{00}}{\partial y^{2}}+\alpha \frac{\partial^{2} u_{01}}{\partial y^{2}}-\alpha y \frac{\partial^{2} u_{00}}{\partial y^{2}}-\alpha^{2} y \frac{\partial^{2} u_{01}}{\partial y^{2}}-\alpha \frac{\partial}{\partial y} u_{00}-\alpha^{2} \frac{\partial}{\partial y} u_{01}-R e i \omega u_{00}-M^{2} u_{00}-\frac{1}{D a} u_{00}+$

$\frac{\alpha y}{D a} u_{00}-R e i \omega \alpha u_{01}-M^{2} \alpha u_{01}-\frac{\alpha}{D a} u_{01}+\frac{\alpha^{2} y}{D a} u_{01}+\frac{R e}{F r} \sin (\delta) e^{-i \omega t}=-\lambda$

By substituting equation (22) into equation (23), with boundary conditions $u_{0}(0)=u_{0}(1)=0$, then equating the like powers of $\alpha$, we obtain the following results presented in the forthcoming subsections:

\section{$i$ - Zeros-order $\operatorname{system}\left(\alpha^{0}\right)$}


$\frac{\partial^{2} u_{00}}{\partial y^{2}}-\left(\operatorname{Re} i \omega+M^{2}+\frac{1}{D a}\right) u_{00}=-\left(\lambda+\frac{R e}{F r} \sin (\delta) e^{-i \omega t}\right)$

The associated boundary conditions are: $u_{00}(0)=u_{00}(1)=0$

$i i$ - First - order $\operatorname{system}\left(\alpha^{1}\right)$

$\frac{\partial^{2} u_{01}}{\partial y^{2}}-\left(\operatorname{Re} i \omega+M^{2}+\frac{1}{D a}\right) u_{01}=y \frac{\partial^{2} u_{00}}{\partial y^{2}}+\frac{\partial}{\partial y} u_{00}-\frac{y}{D a} u_{00}$

The associated boundary conditions are: $u_{01}(0)=u_{01}(1)=0$.

From the solution of equations (24) and (25), and the resulting substitution in equation (20) after substitution in equation (22), we obtain the solution of the equation of motion in the case of Poiseuille flow

$u(y, t)=\left(\begin{array}{l}\frac{E}{A}+\left(-\frac{E}{A\left(1+e^{\sqrt{A}}\right)}\right) e^{\sqrt{A} y}+\left(-\frac{E e^{\sqrt{A}}}{A\left(1+e^{\sqrt{A}}\right)}\right) e^{-\sqrt{A} y}+ \\ \alpha\left(-\frac{K}{A}+e^{\sqrt{A} y}\left(\frac{K}{A\left(1+e^{\sqrt{A}}\right)}\right)+e^{-\sqrt{A} y}\left(\frac{e^{\sqrt{A}} K}{A\left(1+e^{\sqrt{A}}\right)}\right)\right.\end{array}\right) e^{i \omega t}$

where

$$
\begin{aligned}
& K=y\left(-\frac{e^{\sqrt{A} y} E}{1+e^{\sqrt{A}}}-\frac{e^{\sqrt{A}-\sqrt{A} y} E}{1+e^{\sqrt{A}}}\right)+\left(-\frac{e^{\sqrt{A} y} E}{\left(1+e^{\sqrt{A}}\right) \sqrt{A}}+\frac{e^{\sqrt{A}-\sqrt{A} y} E}{\left(1+e^{\sqrt{A}}\right) \sqrt{A}}\right)-\frac{y}{D a}\left(\frac{E}{A}+\left(-\frac{E}{A\left(1+e^{\sqrt{A}}\right)}\right) e^{\sqrt{A} y}+\right. \\
& \left.\left(-\frac{E e^{\sqrt{A}}}{A\left(1+e^{\sqrt{A}}\right)}\right) e^{-\sqrt{A} y}\right)
\end{aligned}
$$

\subsubsection{Couette flow}

Assume here that the flow channel is two parallel plates, the lower plate is at rest and the upper plate is moving with the velocity $U_{0}$. The boundary conditions for the problem in the nondimensional form are:

$u_{0}(0)=0, u_{0}(1)=U_{0} e^{-i \omega t}$

We obtain the solution of the equation of motion in the case of Couette flow.

$$
u(y, t)=e^{i \omega t}\left(\begin{array}{c}
\frac{E}{A}+e^{\sqrt{A} y}\left(-\frac{e^{-i t \omega}\left(-e^{i t \omega} E+e^{\sqrt{A}+i t \omega} E-e^{\sqrt{A}} A U_{0}\right)}{\left(-1+e^{2 \sqrt{A}}\right) A}\right)+ \\
e^{-\sqrt{A} y}\left(-\frac{e^{\sqrt{A}-i t \omega}\left(-e^{i t \omega} E+e^{\sqrt{A}+i t \omega} E+A U_{0}\right)}{\left(-1+e^{2 \sqrt{A}}\right) A}\right)+ \\
\alpha\left(-\frac{K}{A}+e^{\sqrt{A} y}\left(\frac{K}{A\left(1+e^{\sqrt{A}}\right)}\right)+e^{-\sqrt{A} y}\left(\frac{e^{\sqrt{A}} K}{A\left(1+e^{\sqrt{A}}\right)}\right)\right)
\end{array}\right)
$$

where

$$
\begin{aligned}
& K= \\
& y\left(-\frac{e^{\sqrt{A}-\sqrt{A} y-i t \omega}\left(A U_{0}-e^{i t \omega} E+e^{\sqrt{A}+i t \omega} E\right)}{-1+e^{2 \sqrt{A}}}+\frac{e^{\sqrt{A} y-i t \omega}\left(-e^{\sqrt{A}} A U_{0}-e^{i t \omega} E+e^{\sqrt{A}+i t \omega} E\right)}{-1+e^{2 \sqrt{A}}}\right)+
\end{aligned}
$$


$\left(\frac{e^{\sqrt{A}-\sqrt{A} y-i t \omega}\left(A U_{0}-e^{i t \omega} E+e^{\sqrt{A}+i t \omega} E\right)}{\left(-1+e^{2 \sqrt{A}}\right) \sqrt{A}}+\frac{e^{\sqrt{A} y-i t \omega}\left(-e^{\sqrt{A}} A U_{0}-e^{i t \omega} E+e^{\sqrt{A}+i t \omega} E\right)}{\left(-1+e^{2 \sqrt{A}}\right) \sqrt{A}}\right)-$
$\frac{y}{\mathrm{Da}}\left(\frac{E}{A}+e^{\sqrt{A} y}\left(\frac{e^{-i t \omega}\left(-e^{\sqrt{A}} A U_{0}-e^{i t \omega} E+e^{\sqrt{A}+i t \omega} E\right)}{\left(-1+e^{2 \sqrt{A}}\right) A}\right)+e^{-\sqrt{A} y}\left(-\frac{e^{\sqrt{A}-i t \omega}\left(A U_{0}-e^{i t \omega} E+e^{\sqrt{A}+i t \omega} E\right)}{\left(-1+e^{2 \sqrt{A}}\right) A}\right)\right)$.

And $E=\lambda+\frac{R e}{F r} \operatorname{Sin}(\delta) e^{-i \omega t}, A=\left(M^{2}+R e i \omega+\frac{1}{D a}\right)$ in two cases "Poiseuille flow and Couette flow".

\subsection{Solution of the temperature equation}

We will find a solution to the equation of temperature (15) for two types of flows "Poiseuille and Couette". The non-dimensional boundary conditions for heat equation $(15)$ are $\theta(0)=0, \theta(1)=$ 1. To solve the heat equation (15), let

$\theta(y, t)=\theta_{0}(y) e^{i \omega t}$

where $\omega$ is a frequency of the oscillation.

Substituting equation (28) into equation (15), we have

$$
\begin{aligned}
& P e \frac{\partial}{\partial t}\left(\theta_{0}(y) e^{i \omega t}\right)=\frac{\partial^{2}}{\partial y^{2}}\left(\theta_{0}(y) e^{i \omega t}\right)+N^{2}\left(\theta_{0}(y) e^{i \omega t}\right)+\mu(y) B r\left(\frac{\partial u}{\partial y}\right)^{2}+B r B n\left(\frac{\partial u}{\partial y}\right) \\
& \Longrightarrow P e \frac{\partial}{\partial t}\left(\theta_{0}(y) e^{i \omega t}\right)=\frac{\partial^{2}}{\partial y^{2}}\left(\theta_{0}(y) e^{i \omega t}\right)+N^{2}\left(\theta_{0}(y) e^{i \omega t}\right)+e^{-\alpha y} B r\left(\frac{\partial u}{\partial y}\right)^{2}+\operatorname{BrBn}\left(\frac{\partial u}{\partial y}\right)
\end{aligned}
$$

implies that

$\frac{\partial^{2} \theta_{0}(y)}{\partial y^{2}}+\left(N^{2}-i \omega P e\right) \theta_{0}(y)=-e^{-i \omega t}\left(e^{-\alpha y} B r\left(\frac{\partial u}{\partial y}\right)^{2}+B r B n\left(\frac{\partial u}{\partial y}\right)\right)$

The solution of equation (29) with boundary conditions $\theta_{0}(0)=0, \theta_{0}(1)=e^{-i \omega t}$, is divided into two parts according to the type of flow "Poiseuille flow or Couette flow" and thus we get the solution.

The value of the solution is very large and we will discuss it in the drawings.

\subsection{Solution of the concentration equation:}

To solve the concentration equation (16) with boundary conditions: $\Phi(0)=0, \Phi(1)=1$

Let

$\Phi(y, t)=\Phi_{0}(y) e^{i \omega t}$

Substituting equations (30) and (28) by equation (16), results in

$\frac{\partial}{\partial t}\left(\Phi_{0}(y) e^{i \omega t}\right)=\frac{1}{S_{c}} \frac{\partial^{2}}{\partial y^{2}}\left(\Phi_{0}(y) e^{i \omega t}\right)-K_{r}\left(\Phi_{0}(y) e^{i \omega t}\right)+\operatorname{Sr} \frac{\partial^{2}}{\partial y^{2}}\left(\theta_{0}(y) e^{i \omega t}\right)$

Implies that

$\frac{\partial^{2} \Phi_{0}}{\partial y^{2}}-S c\left(K_{r}+i \omega\right) \Phi_{0}+S c S r \frac{\partial^{2} \theta_{0}}{\partial y^{2}}=0$ 
The solution of equation (31) with boundary conditions $\Phi_{0}(0)=0, \Phi_{0}(1)=e^{-i \omega t}$, is divided into two parts according to the type of flow "Poiseuille flow or Couette flow" and thus we get the solution.

The value of the solution is very large and we will discuss it in the drawings.

\section{Numerical Results and Discussion:}

This section discussed the influence of temperature and concentration on the magnetohydrodynamics oscillatory flow of Bingham fluid with variable viscosity through a porous medium for Poiseuille flow and Couette flow. use the MATHEMATICA-12 program, to discussed graphically all solutions obtained (velocity, temperature and concentration) of the fluid under variations of different parameters relevant, significant results are presented in figures (2-39), through area $0 \leq \mathrm{y} \leq 1$ which is the width of the flow channel.

Based on equations (26) and (27), the velocity profile appears in figures (2-10), where each figure is divided into two figures, (a) representing Poiseuille flow, and (b) representing Couette flow. Fig 2 shows the velocity profile $u$ decreases with the increasing $t$. Fig 3 illustrates the influence $\lambda$ on the velocity profiles vs. $y$. It is found by the increasing $\lambda$ the velocity profiles function $u$ (decreases). Fig 4 shows that $u$ rising up by the increasing $D a$. Fig 5 shows that $u$ increases with the increase of $R e$. Fig 6 shows that $u$ go down with increasing $M$. Fig 7 illustrates the influence $(\omega)$ on the velocity profiles vs. $y$. It is found by the increasing $(\omega)$ the velocity profile decreases. Fig 8 show that the velocity increasing with the increasing of $\alpha$ when $0.3<y \leq$ 1 , and decreases when $0 \leq y<0.3$ in Poiseuille flow, while in Couette flow the velocity decreases with the increaseing $\alpha$. Fig 9 shows that $u$ rising up by the increasing $\delta$. Fig 10 shows that $u$ decreases with the increase of Fr. We noticed from drawings 2-10 that the fluid flow velocity in the case of Couette flow) is more than that of (Poiseuille flow) due to the movement of the upper wall of the channel at a constant velocity $\left(U_{0}=0.3\right)$, which is why the highest value of the fluid velocity in Poiseuille flow is in the middle of the flow channel, while the highest value of the speed in Couette flow is at the upper wall.

Based on equation (29), the temperature profile appears in figures (11-23), where each figure is divided into two figures, (a) representing Poiseuille flow, and (b) representing Couette flow. Fig 11 shows the temperature profile decreases with the increasing $M$. Fig 12 show us that with the increasing of $\omega$ the temperature decreases in Poiseuille flow, while in Couette flow the temperature profile increasing with the increasing $\omega$. Fig 13 show us that with the increasing of $B n$ the temperature decreases when $0.5<y \leq 1$, and increases when $0 \leq y<0.5$ in Poiseuille flow, while in Couette flow the temperature profile increasing with the increasing Bn. Fig 14 shows the temperature profile increasing with the increasing $B r$. Fig 15 shows the temperature profile decreases with the increasing $P e$. Figs 16 and 17 show us that with the increasing of $N$ and $D a$ the temperature increasing. Figs 18 and 19 show us that with the increasing of $R e$ and $\lambda$ the temperature increasing. Figure 20 show shows the temperature profile decreases with the increasing $t$ in Poiseuille flow, while in Couette flow the temperature decreases when $0.7<y \leq$ 1 , and increasing when $0 \leq y<0.7$. Figure 21 show us that with the increasing of $\alpha$ the temperature increasing when $0.4<y \leq 1$, and decreases when $0 \leq y<0.4$ in Poiseuille flow, while in Couette flow the temperature profile decreases with the increasing $\alpha$. Figure 22 shows the temperature profile increasing with the increasing $\delta$. Figure 23 shows the temperature profile decreases with the increasing $\mathrm{Fr}$. 
Based on equation (31), the concentration profile appears in figures (24-39), where each figure is divided into two figures, (a) representing Poiseuille flow, and (b) representing Couette flow. Figure 24 shows the concentration profile decreases with the increasing $S c$ in Poiseuille flow, while in Couette flow the concentration increasing when $0.5<y \leq 1$, and decreases when $0 \leq y<0.5$. Figure 25 shows the concentration profile $\Phi$ increasing with the increasing $\omega$. Figure 26 shows the concentration profile $\Phi$ decreases with the increasing $N$. Figure 27 shows the concentration profile decreases with the increasing $\mathrm{Sr}$ in Poiseuille flow, while in Couette flow the concentration increasing when $0.5<y \leq 1$, and decreases when $0 \leq y<0.5$. Figure 28 shows the concentration profile decreases with the increasing $M$ in Poiseuille flow, while in Couette flow the concentration decreases when $0.8<y \leq 1$, and increasing when $0 \leq y<0.8$. Figure 29 shows the concentration profile $\Phi$ increasing with the increasing $P e$. Figure 30 shows the concentration profile decreases with the increasing $\mathrm{Br}$ in Poiseuille flow, while in Couette flow the concentration increasing when $0.5<y \leq 1$, and decreases when $0 \leq y<0.5$. Figure 31 show that with the increasing of $B n$ the concentration increases when $0.5<y \leq 1$, and decreases when $0 \leq y<0.5$. Figure 32 shows the concentration profile $\Phi$ increasing with the increasing $t$. Figure 33 shows the concentration profile $\Phi$ decreases with the increasing $\delta$. Figure 34 shows the concentration profile $\Phi$ increasing with the increasing $F r$. Figure 35 show us that with the increasing of $\alpha$ the concentration decreases when $0.5<y \leq 1$, and increasing when $0 \leq y<0.5$, in Poiseuille flow, while in Couette flow the concentration increasing. Figure 36 show shows the concentration profile decreases with the increasing $R e$. Figure 37 shows the concentration profile $\Phi$ increasing with the increasing $D a$ in Poiseuille flow, while in Couette flow the concentration decreases. Figure 38 shows the concentration profile $\Phi$ decreases with the increasing $\lambda$. Figure 39 shows the concentration profile decreases with the increasing $K_{r}$ in Poiseuille flow, while in Couette flow the concentration increasing when $0.5<y \leq 1$, and decreases when $0 \leq y<0.5$.
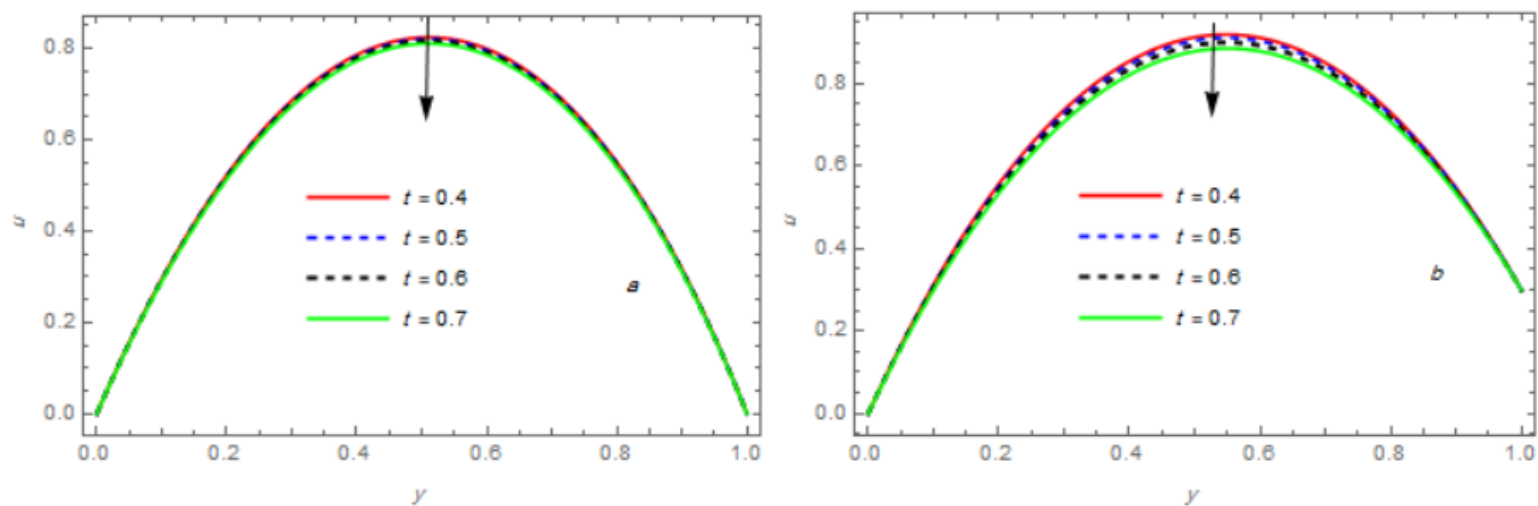

Fig 2 Velocity profile with different values $t$ for (a) Poiseuille flow and (b) Couette flow, with $D a=0.8, M=1, \omega=1, \lambda=1, \alpha=0.05, \operatorname{Re}=1, \xi=\frac{\pi}{4}, \delta=\frac{\pi}{4}, U_{0}=0.3, F r=0.3$. 

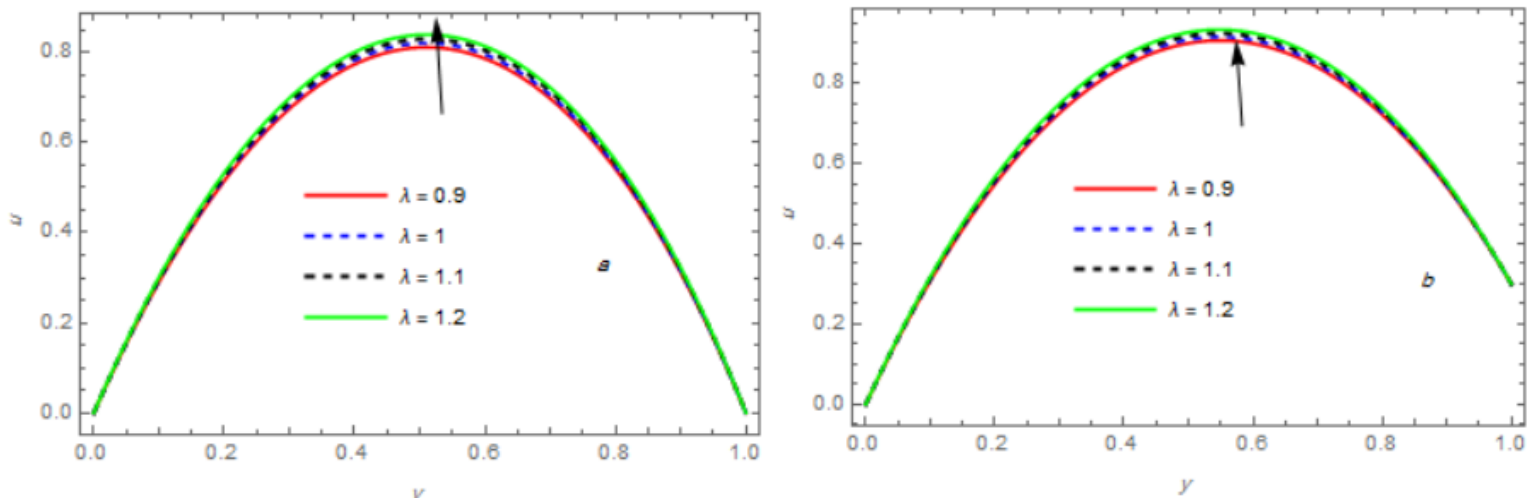

Fig 3 Velocity profile with different values $\lambda$ for (a) Poiseuille flow and (b) Couette flow, with $D a=0.8, M=1, \omega=1, \alpha=0.05, \operatorname{Re}=1, \xi=\frac{\pi}{4}, \delta=\frac{\pi}{4}, t=0.5, U_{0}=0.3, F r=0.3$.
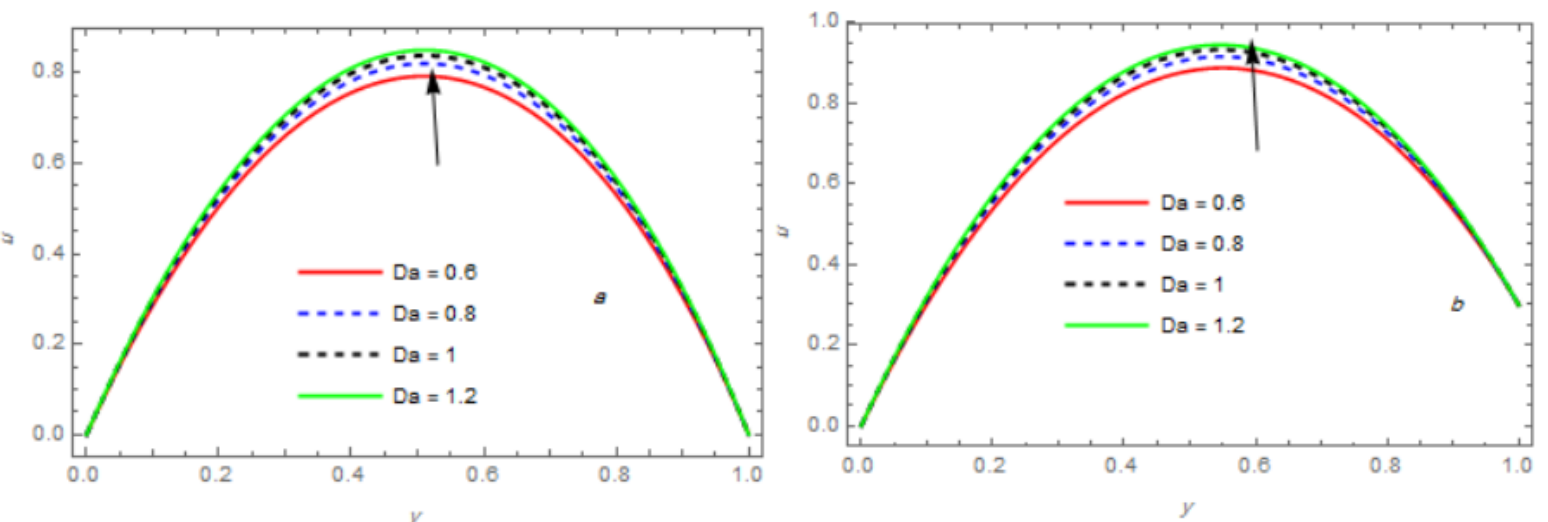

Fig 4 Velocity profile with different values $D a$ for (a) Poiseuille flow and (b) Couette flow, with $M=1, \omega=1, \lambda=1, \alpha=0.05, \operatorname{Re}=1, \xi=\frac{\pi}{4}, \delta=\frac{\pi}{4}, U_{0}=0.3, t=0.5, F r=0.3$.
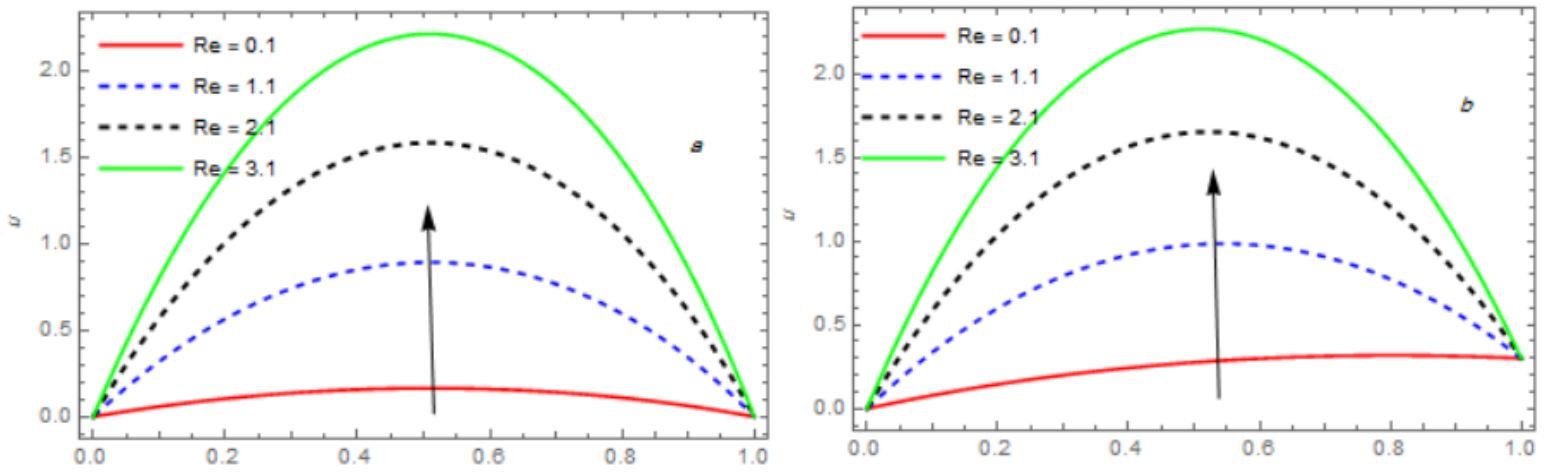

Fig 5 Velocity profile with different values Re for (a) Poiseuille flow and (b) Couette flow, with $D a=0.8, M=1, \omega=1, \lambda=1, \alpha=0.05, \xi=\frac{\pi}{4}, \delta=\frac{\pi}{4}, U_{0}=0.3, t=0.5, F r=0.3$. 

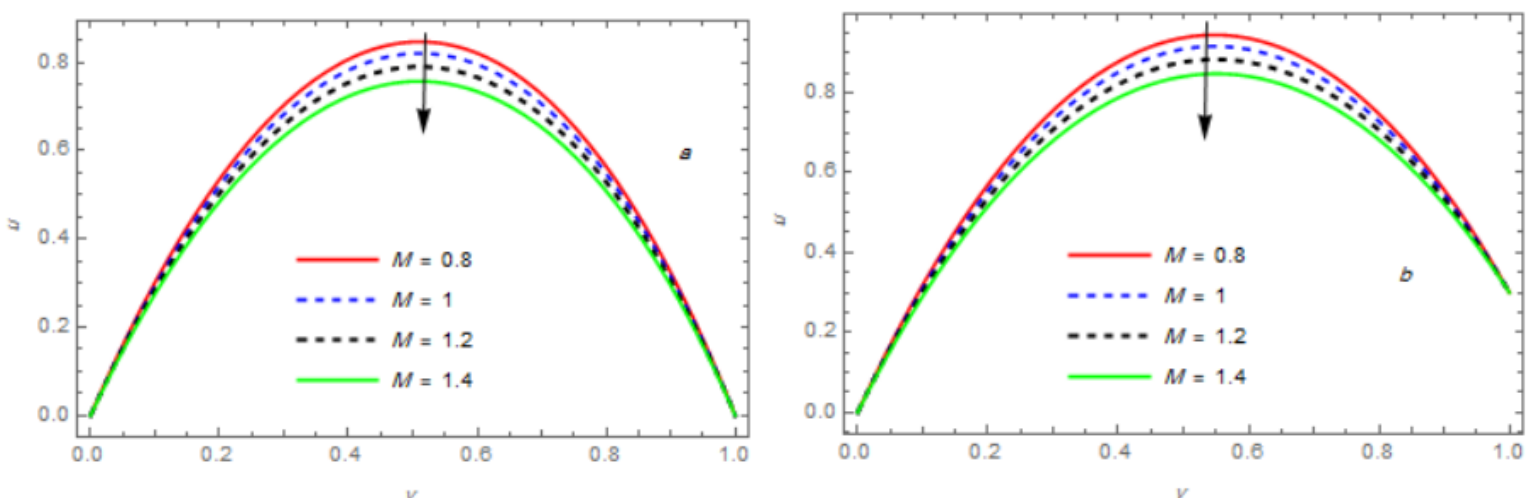

Fig 6 Velocity profile with different values $M$ for (a) Poiseuille flow and (b) Couette flow, with $D a=0.8, \omega=1, \lambda=1, \alpha=0.05, \operatorname{Re}=1, \xi=\frac{\pi}{4}, \delta=\frac{\pi}{4}, U_{0}=0.3, t=0.5, F r=0.3$.
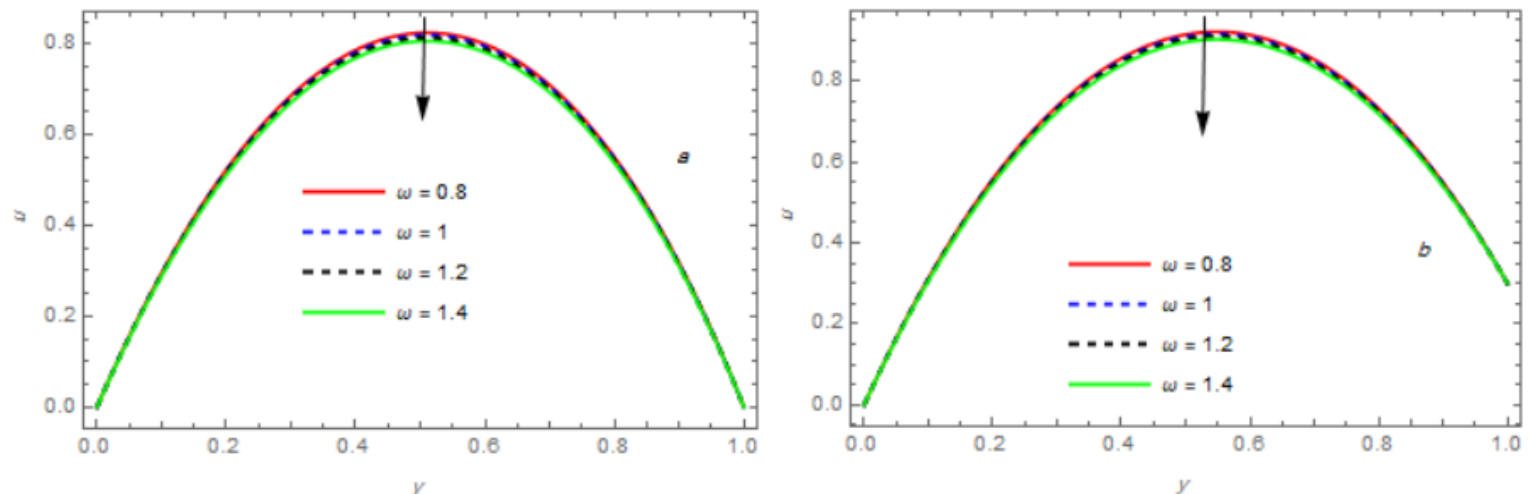

Fig 7 Velocity profile with different values $\omega$ for (a) Poiseuille flow and (b) Couette flow, with $D a=0.8, M=1, \lambda=1, \alpha=0.05, R e=1, \xi=\frac{\pi}{4}, \delta=\frac{\pi}{4}, U_{0}=0.3, t=0.5, F r=0.3$.
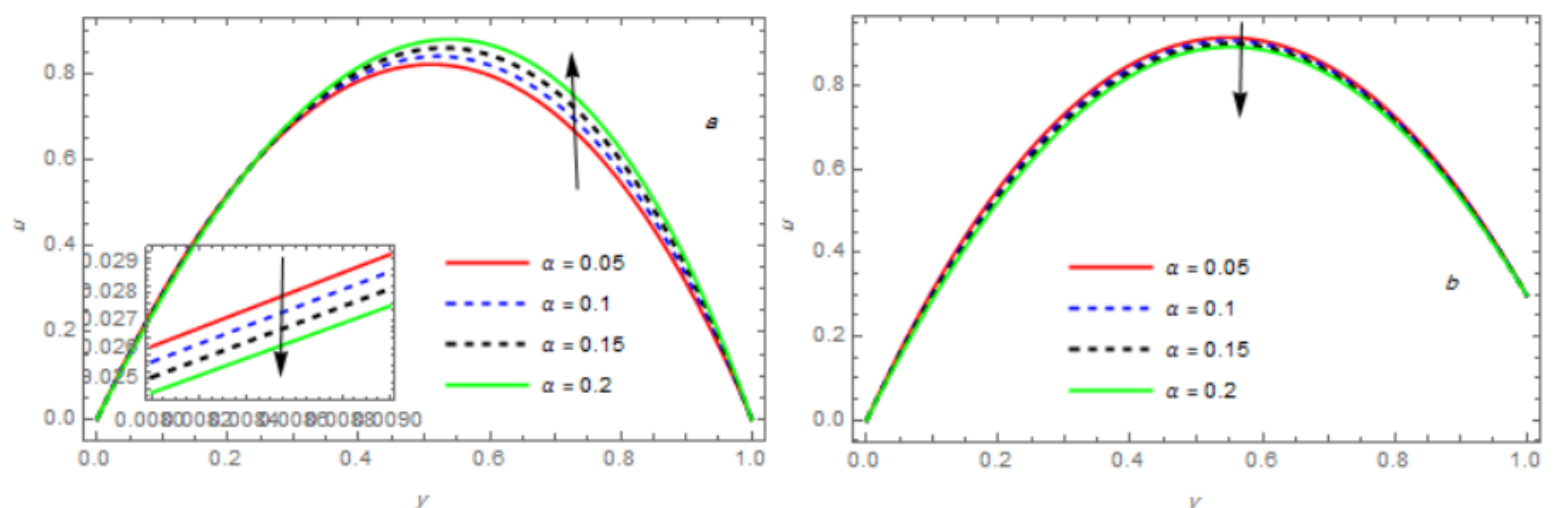

Fig 8 Velocity profile with different values $\alpha$ for (a) Poiseuille flow and (b) Couette flow, with $D a=0.8, M=1, \omega=1, \lambda=1, \operatorname{Re}=1, \xi=\frac{\pi}{4}, \delta=\frac{\pi}{4}, U_{0}=0.3, t=0.5, F r=0.3$. 

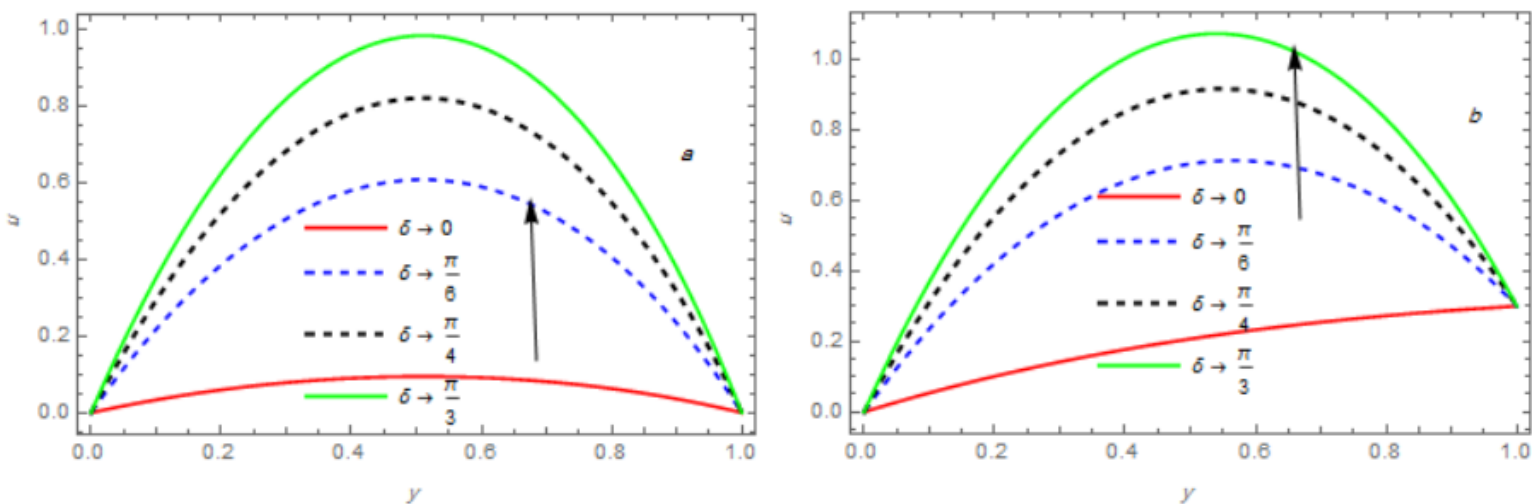

Fig 9 Velocity profile with different values $\delta$ for (a) Poiseuille flow and (b) Couette flow, with $D a=0.8, M=1, \omega=1, \lambda=1, \operatorname{Re}=1, \xi=\frac{\pi}{4}, U_{0}=0.3, t=0.5, F r=0.3$.
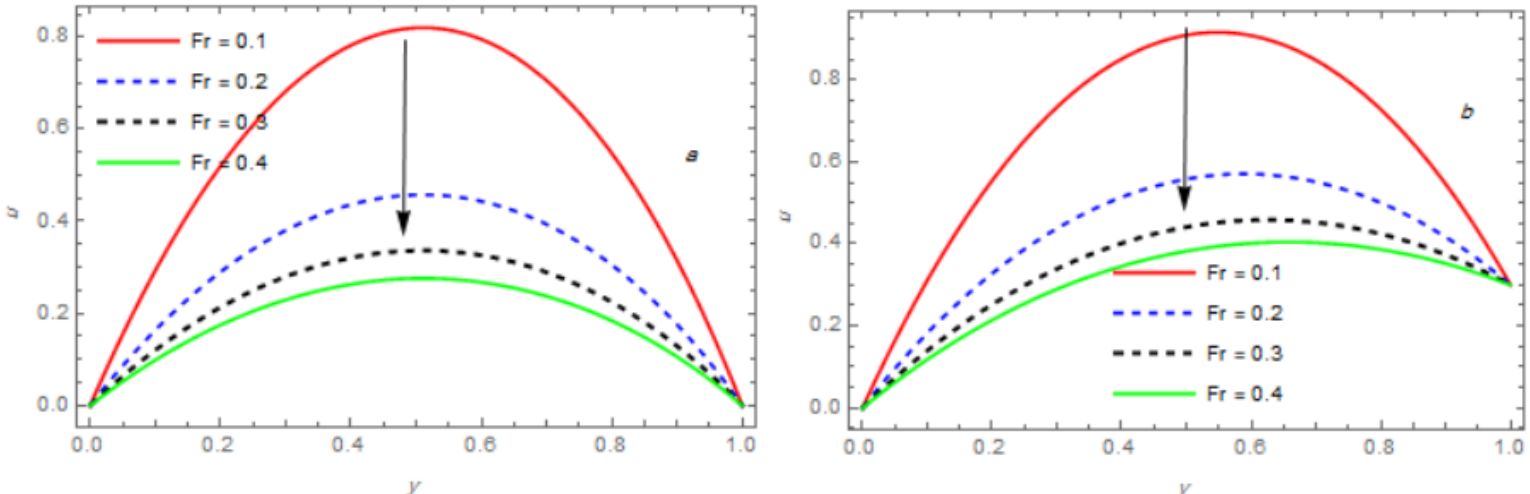

Fig 10 Velocity profile with different values $F r$ for (a) Poiseuille flow and (b) Couette flow, with $D a=0.8, M=1, \omega=1, \lambda=1, R e=1, \xi=\frac{\pi}{4}, \delta=\frac{\pi}{4}, U_{0}=0.3, t=0.5$.
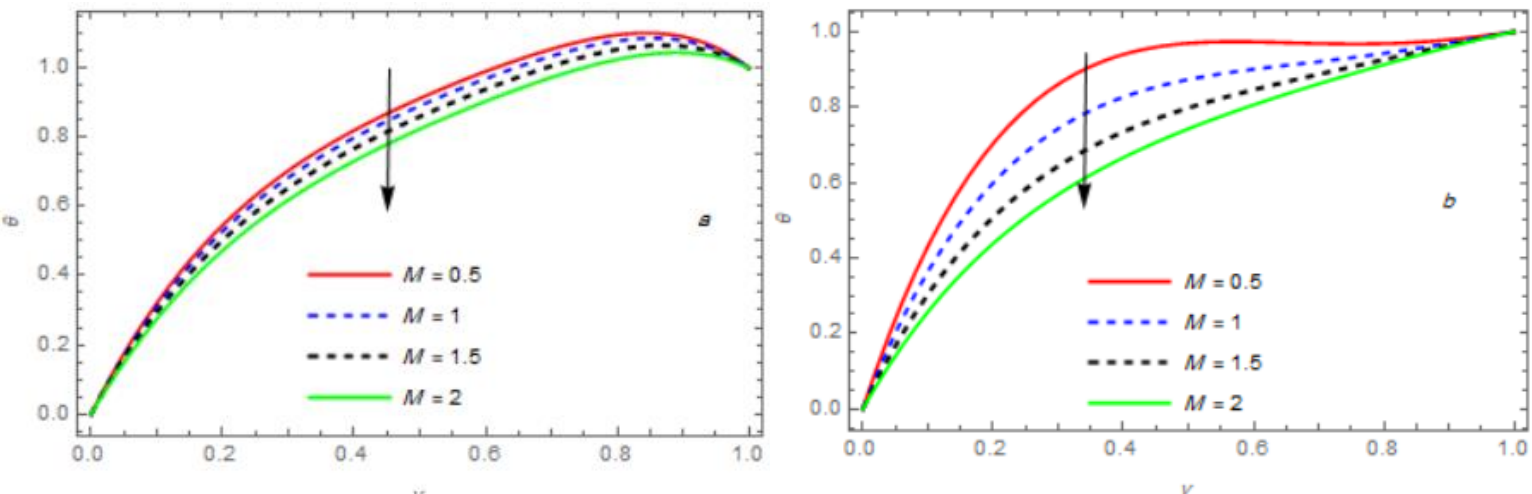

Fig 11 Temperature profile with different values $M$ for (a) Poiseuille flow and (b) Couette flow, with $B n=1, P e=2, B r=1, \alpha=0.05, \omega=1, N=1.25, D a=0.8, \lambda=1, R e=$ $1, \xi=\frac{\pi}{4}, \delta=\frac{\pi}{4}, U_{0}=0.3, t=0.5, \mathrm{Fr}=0.3$. 

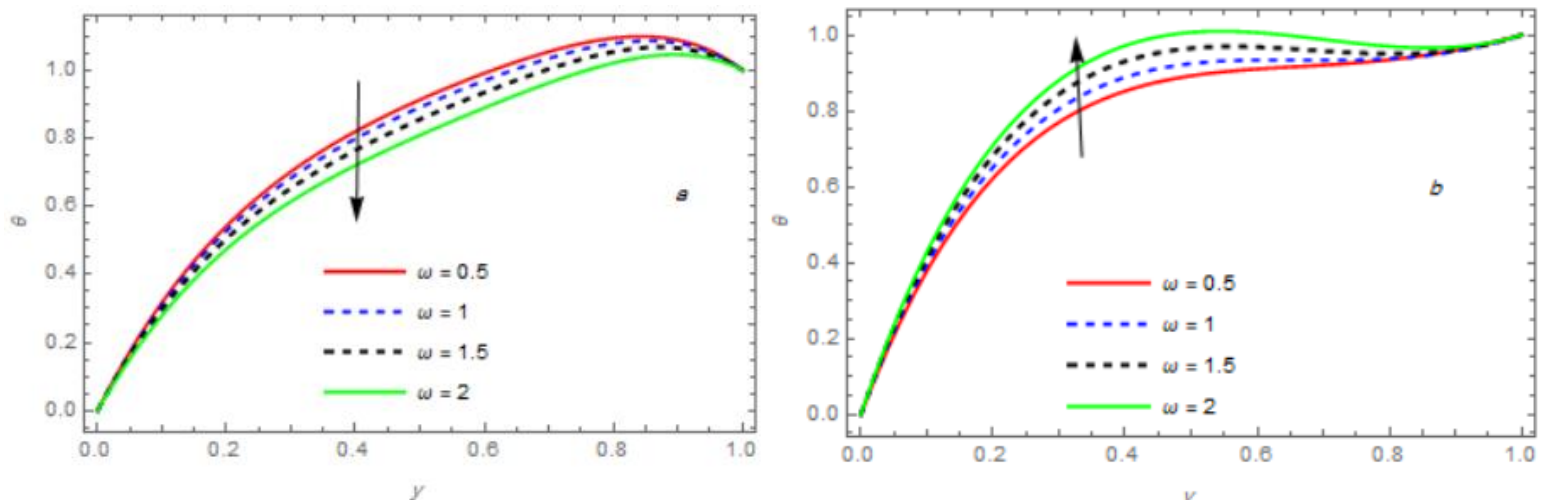

Fig 12 Temperature profile with different values $\omega$ for (a) Poiseuille flow and (b) Couette flow, with $B n=1, P e=2, B r=1, \alpha=0.05, N=1.25, D a=0.8, M=1, \lambda=1, R e=$ $1, \xi=\frac{\pi}{4}, \delta=\frac{\pi}{4}, U_{0}=0.3, t=0.5, F r=0.3$.
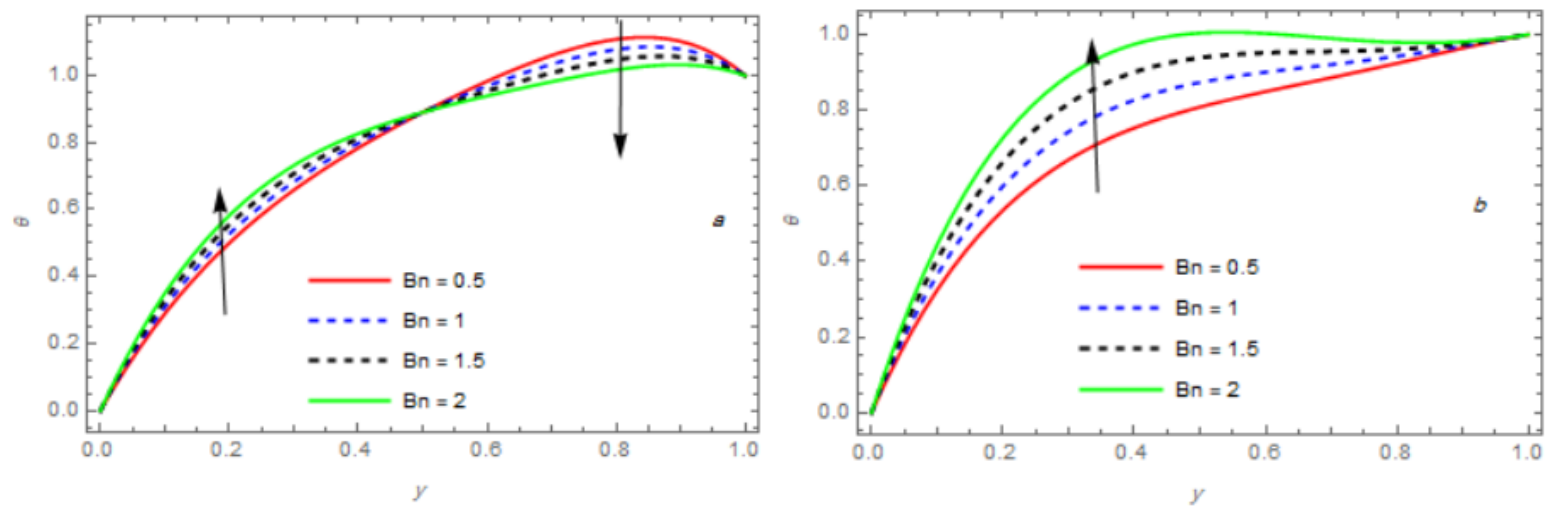

Fig 13 Temperature profile with different values $B n$ for (a) Poiseuille flow and (b) Couette flow, with $\omega=1, P e=2, B r=1, \alpha=0.05, N=1.25, D a=0.8, M=1, \lambda=1, \operatorname{Re}=$ $1, \xi=\frac{\pi}{4}, \delta=\frac{\pi}{4}, U_{0}=0.3, t=0.5, F r=0.3$.
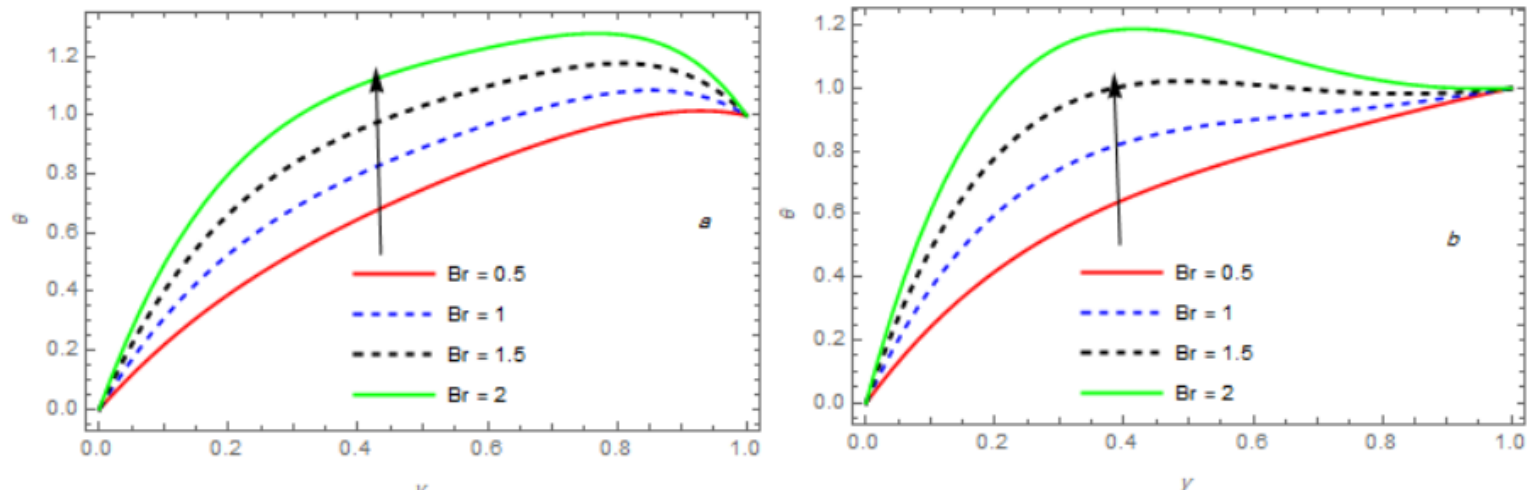

Fig 14 Temperature profile with different values $B r$ for (a) Poiseuille flow and (b) Couette flow, with $\omega=1, P e=2, B n=1, \alpha=0.05, N=1.25, D a=0.8, M=1, \lambda=1, R e=$ $1, \xi=\frac{\pi}{4}, \delta=\frac{\pi}{4}, U_{0}=0.3, t=0.5, \mathrm{Fr}=0.3$. 

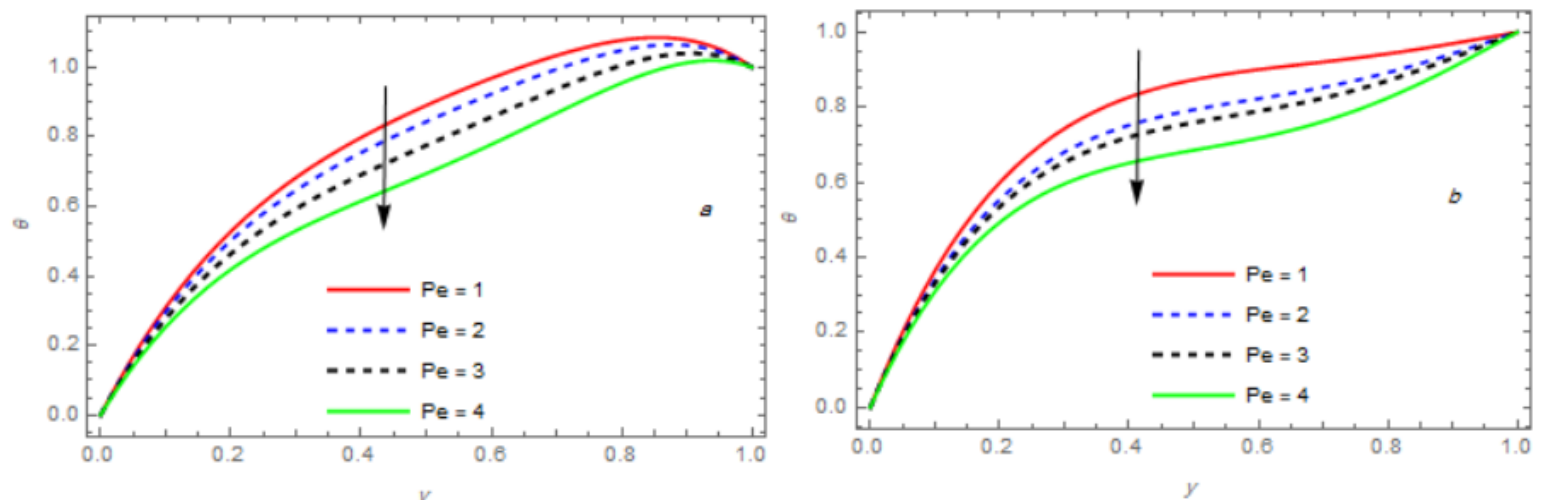

Fig 15 Temperature profile with different values $P e$ for (a) Poiseuille flow and (b) Couette flow, with $\omega=1, B r=1, B n=1, \alpha=0.05, N=1.25, D a=0.8, M=1, \lambda=1, \operatorname{Re}=$ $1, \xi=\frac{\pi}{4}, \delta=\frac{\pi}{4}, U_{0}=0.3, t=0.5, \mathrm{Fr}=0.3$.
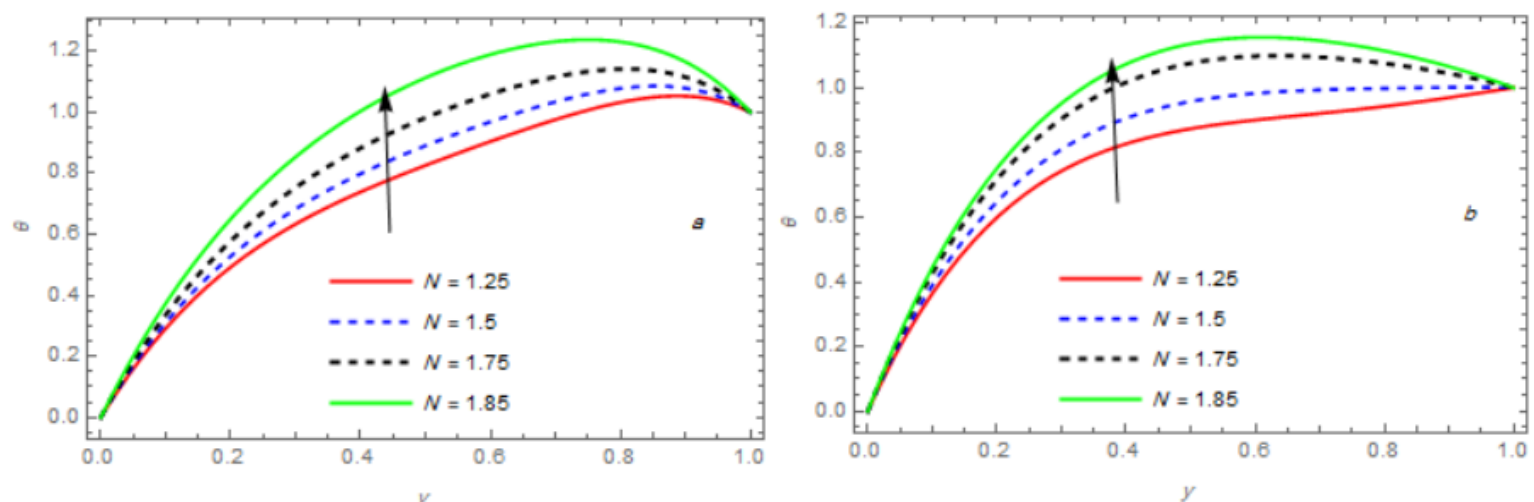

Fig 16 Temperature profile with different values $N$ for (a) Poiseuille flow and (b) Couette flow, with $\omega=1, B r=1, B n=1, \alpha=0.05, P e=2, D a=0.8, M=1, \lambda=1, \operatorname{Re}=1, \xi=\frac{\pi}{4}, \delta=$ $\frac{\pi}{4}, U_{0}=0.3, t=0.5, F r=0.3$.
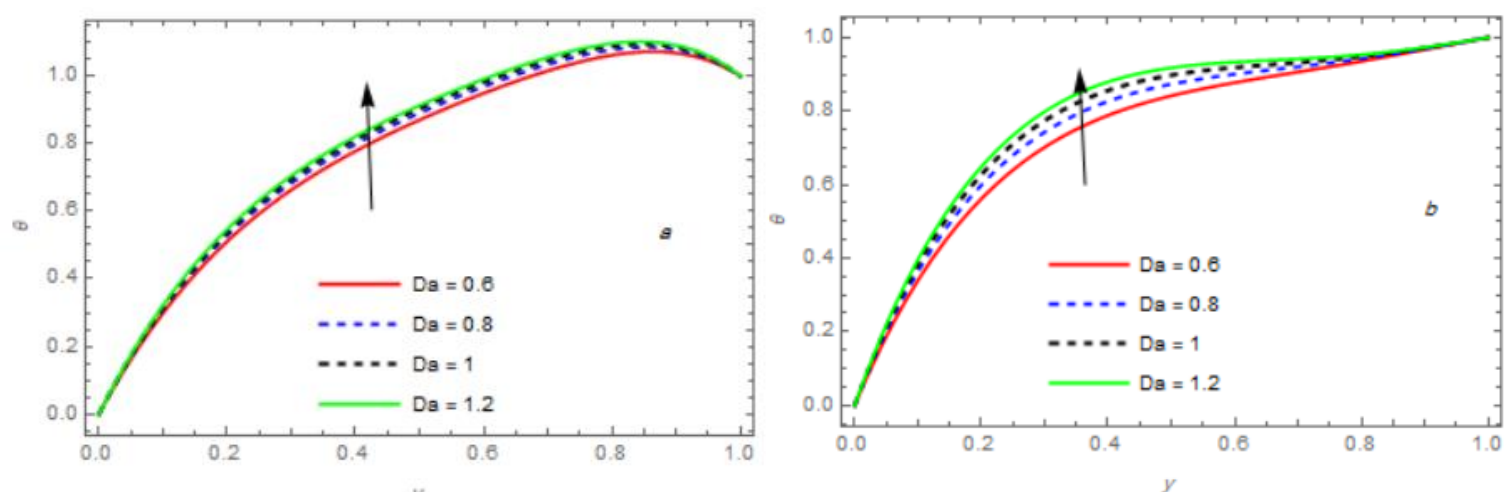

Fig 17 Temperature profile with different values $D a$ for (a) Poiseuille flow and (b) Couette flow, with $\omega=1, B r=1, B n=1, \alpha=0.05, P e=2, N=1.25, M=1, \lambda=1, \operatorname{Re}=$ $1, \xi=\frac{\pi}{4}, \delta=\frac{\pi}{4}, U_{0}=0.3, t=0.5, F r=0.3$. 

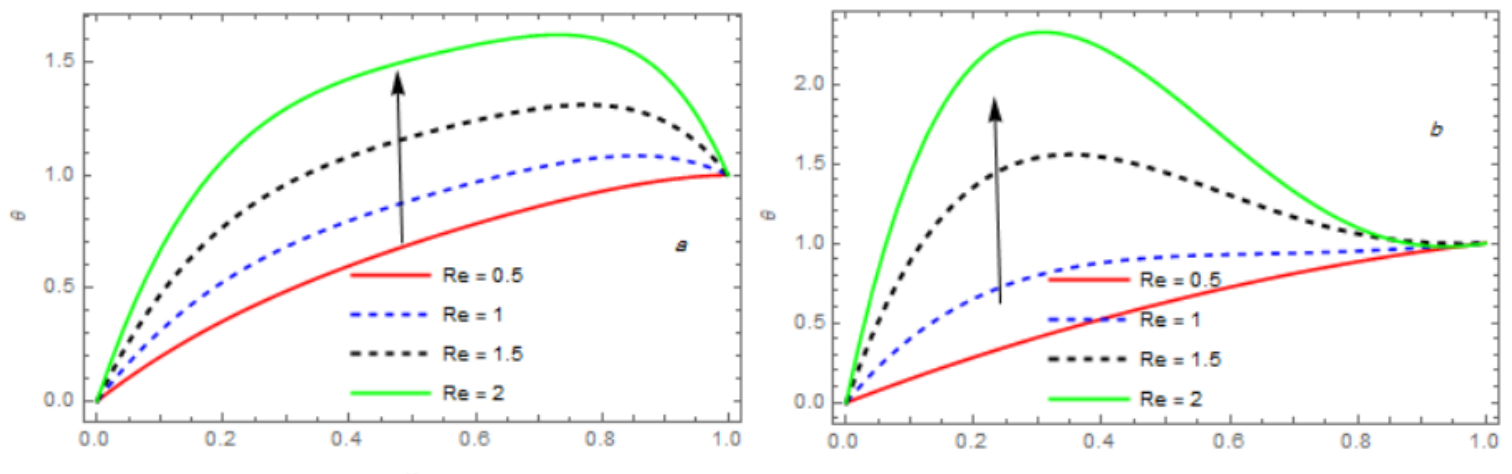

Fig 18 Temperature profile with different values $R e$ for (a) Poiseuille flow and (b) Couette flow, with $\omega=1, B r=1, B n=1, \alpha=0.05, P e=2, D a=0.8, M=1, \lambda=1, N=$ $1.25, \xi=\frac{\pi}{4}, \delta=\frac{\pi}{4}, U_{0}=0.3, t=0.5, F r=0.3$.
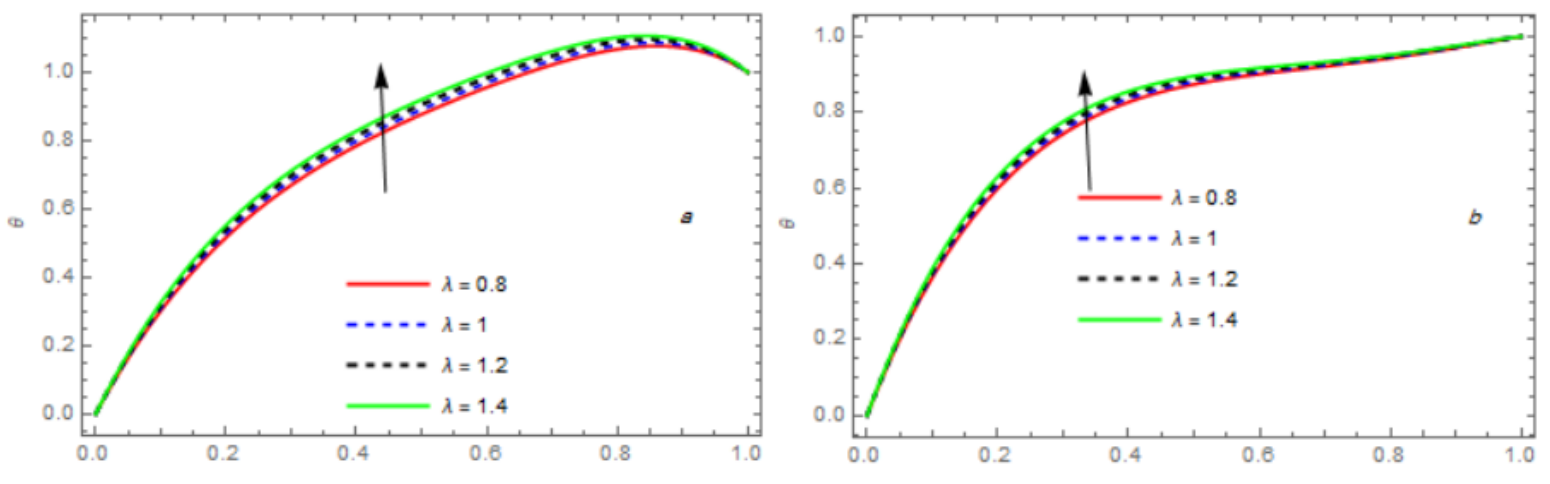

Fig 19 Temperature profile with different values $\lambda$ for (a) Poiseuille flow and (b) Couette flow, with $\omega=1, B r=1, B n=1, \alpha=0.05, P e=2, D a=0.8, M=1, R e=1, N=1.25, \xi=$ $\frac{\pi}{4}, \delta=\frac{\pi}{4}, U_{0}=0.3, t=0.5, F r=0.3$.
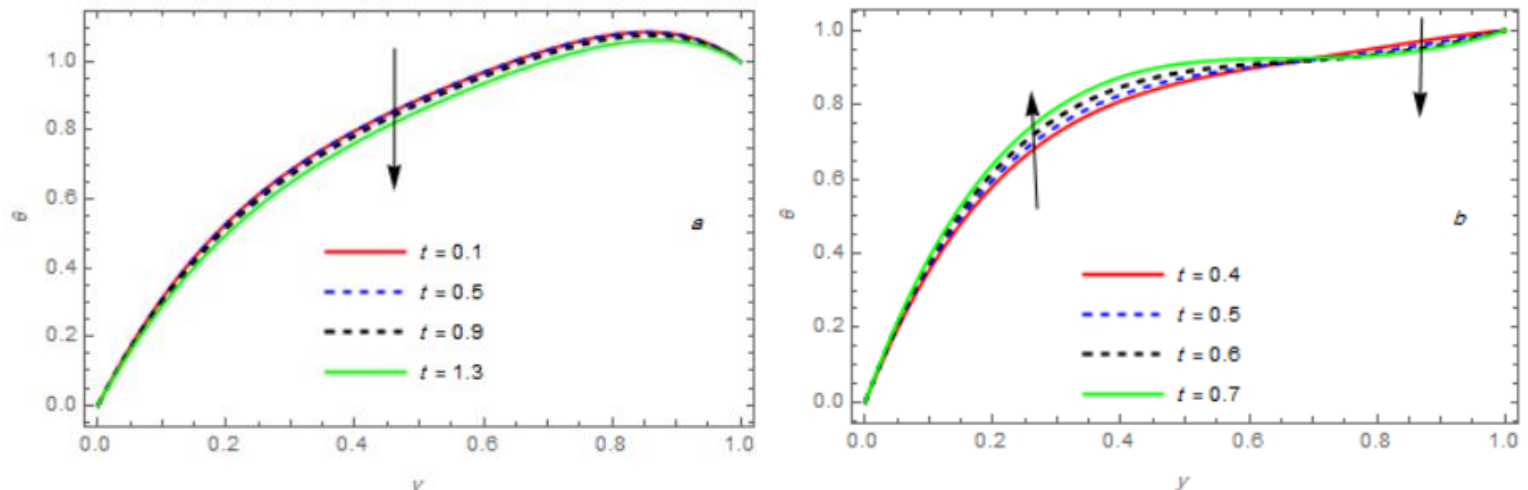

Fig 20 Temperature profile with different values $t$ for (a) Poiseuille flow and (b) Couette flow, with $\omega=1, B r=1, B n=1, \alpha=0.05, P e=2, D a=0.8, M=1, \operatorname{Re}=1, N=1.25, \xi=$ $\frac{\pi}{4}, \delta=\frac{\pi}{4}, U_{0}=0.3, \lambda=1, \mathrm{Fr}=0.3$. 

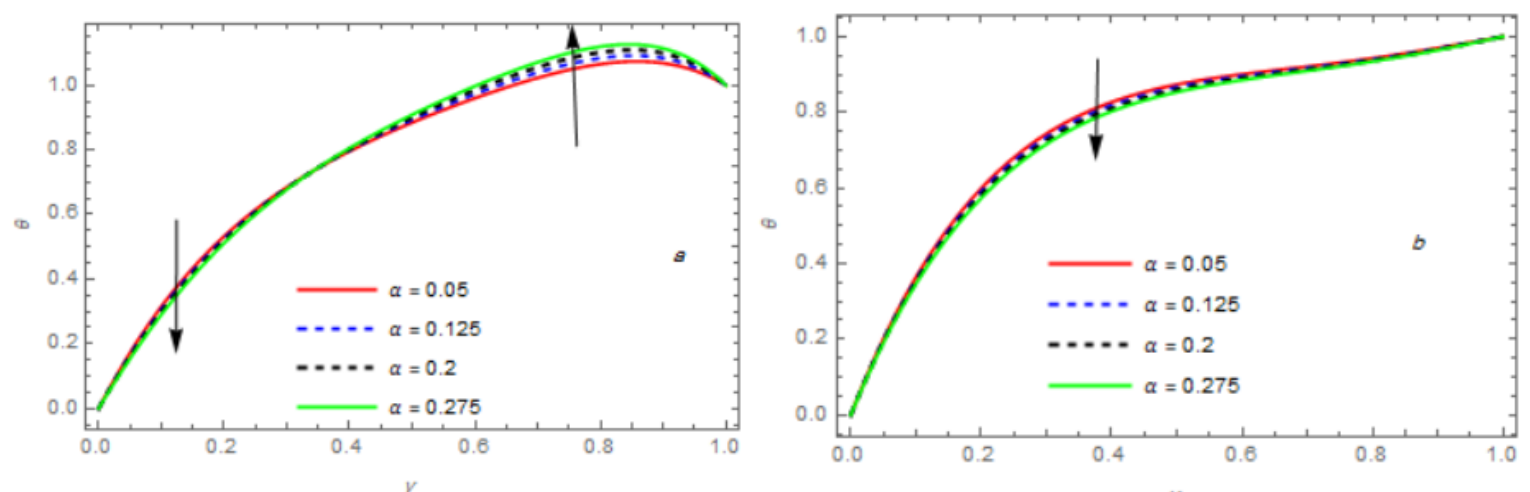

Fig 21 Temperature profile with different values $\alpha$ for (a) Poiseuille flow and (b) Couette flow, with $\omega=1, B r=1, B n=1, t=0.5, P e=2, D a=0.8, M=1, R e=1, N=1.25, \xi=\frac{\pi}{4}, \delta=$ $\frac{\pi}{4}, U_{0}=0.3, \lambda=1, F r=0.3$.
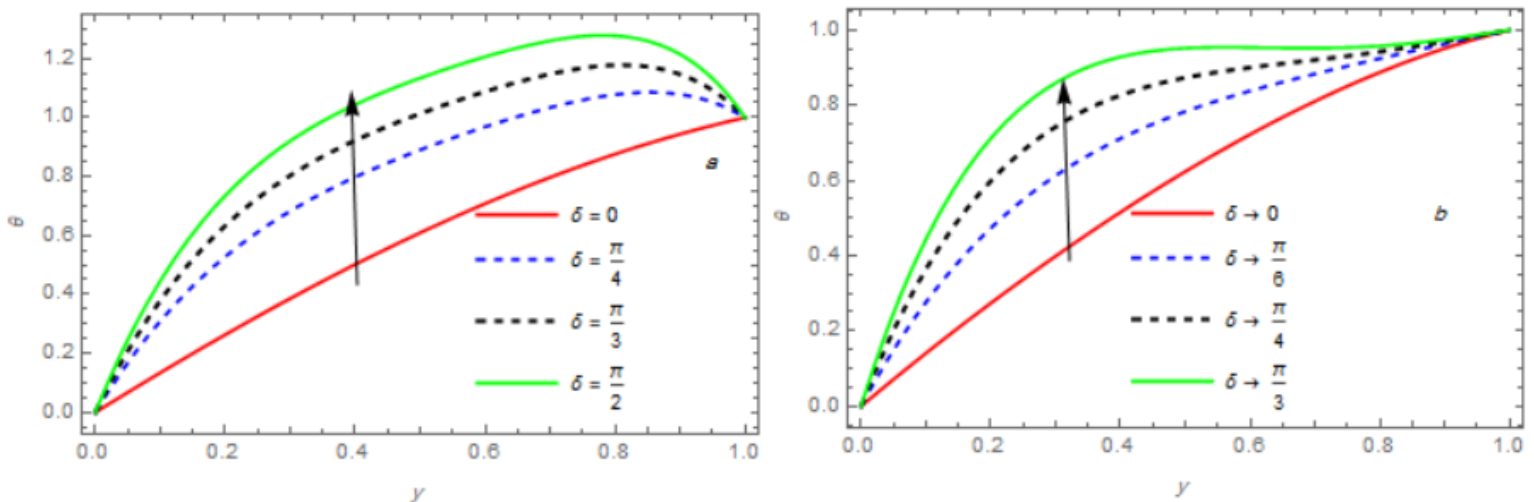

Fig 22 Temperature profile with different values $\delta$ for (a) Poiseuille flow and (b) Couette flow, with $\omega=1, B r=1, B n=1, t=0.5, P e=2, D a=0.8, M=1, R e=1, N=1.25, \xi=$ $\frac{\pi}{4}, U_{0}=0.3, \lambda=1, F r=0.3$.
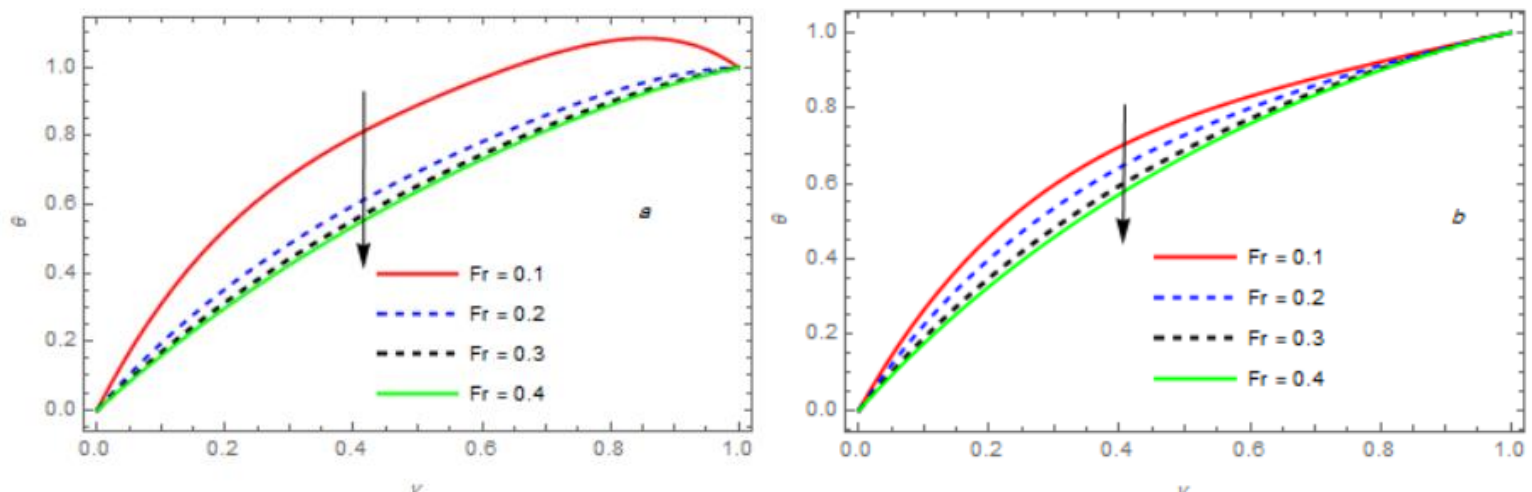

Fig 23 Temperature profile with different values $\mathrm{Fr}$ for (a) Poiseuille flow and (b) Couette flow, with $\omega=1, B r=1, B n=1, t=0.5, P e=2, D a=0.8, M=1, R e=1, N=$ $1.25, \xi=\frac{\pi}{4}, \delta=\frac{\pi}{4}, U_{0}=0.3, \lambda=1$. 

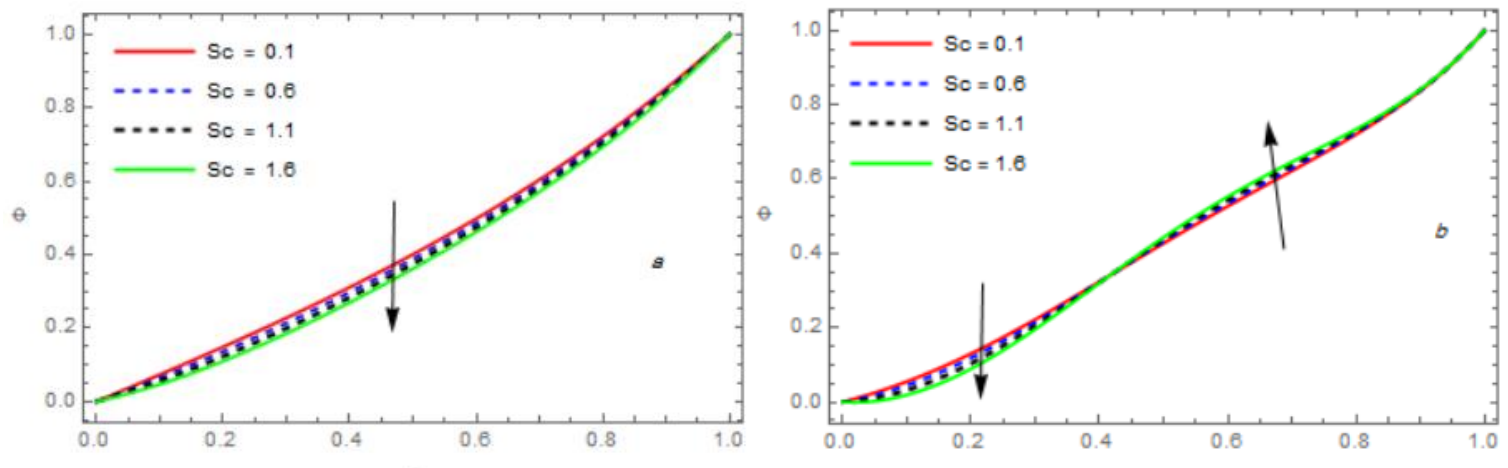

Fig 24 Influence of $S c$ on concentration for $B n=1, P e=2, \alpha=0.05, B r=1, \omega=1, M=$ $1, K_{r}=0.5, S r=0.1, t=0.5, D a=0.8, \lambda=1, R e=1, \xi=\frac{\pi}{4}, \delta=\frac{\pi}{4}, U_{0}=0.3, N=$ $1.25, \mathrm{Fr}=0.3$.
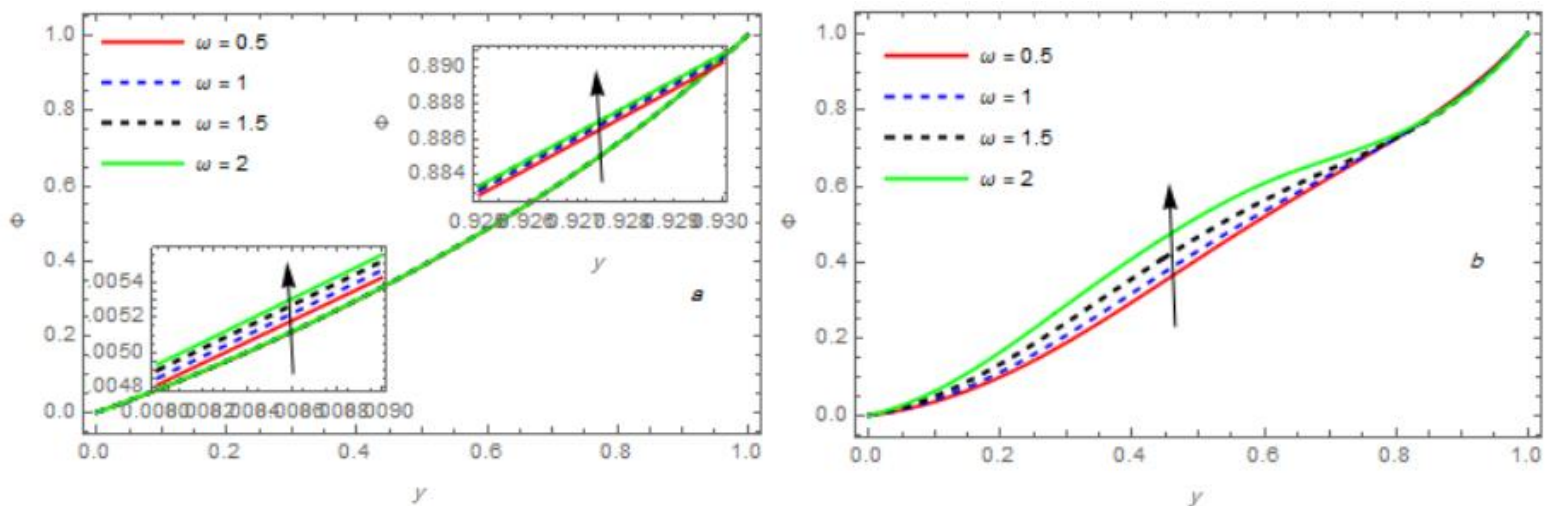

Fig 25 Influence of $\omega$ on concentration for $B n=1, P e=2, \alpha=0.05, B r=1, S c=0.6, M=$ $1, K_{r}=0.5, S r=0.1, t=0.5, D a=0.8, \lambda=1, R e=1, \xi=\frac{\pi}{4}, \delta=\frac{\pi}{4}, U_{0}=0.3, N=$ $1.25, \mathrm{Fr}=0.3$.
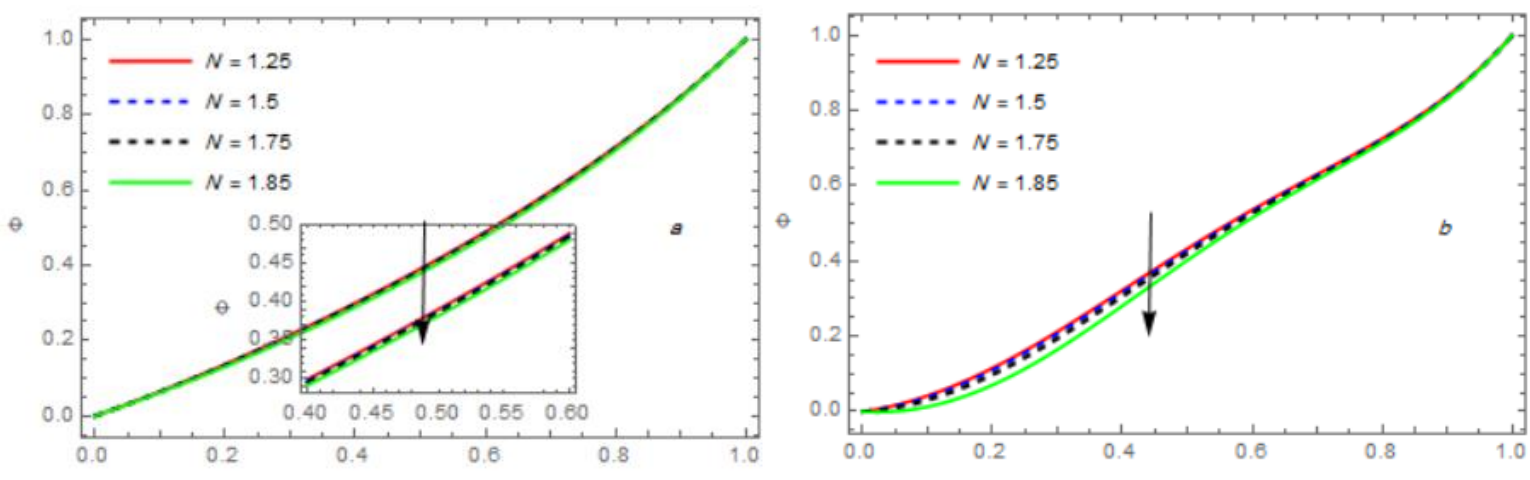

Fig 26 Influence of $N$ on concentration for $B n=1, P e=2, \alpha=0.05, B r=1, \omega=1, M=$ $1, K_{r}=0.5, S r=0.1, t=0.5, D a=0.8, \lambda=1, R e=1, \xi=\frac{\pi}{4}, \delta=\frac{\pi}{4}, U_{0}=0.3, S c=$ $0.6, F r=0.3$. 

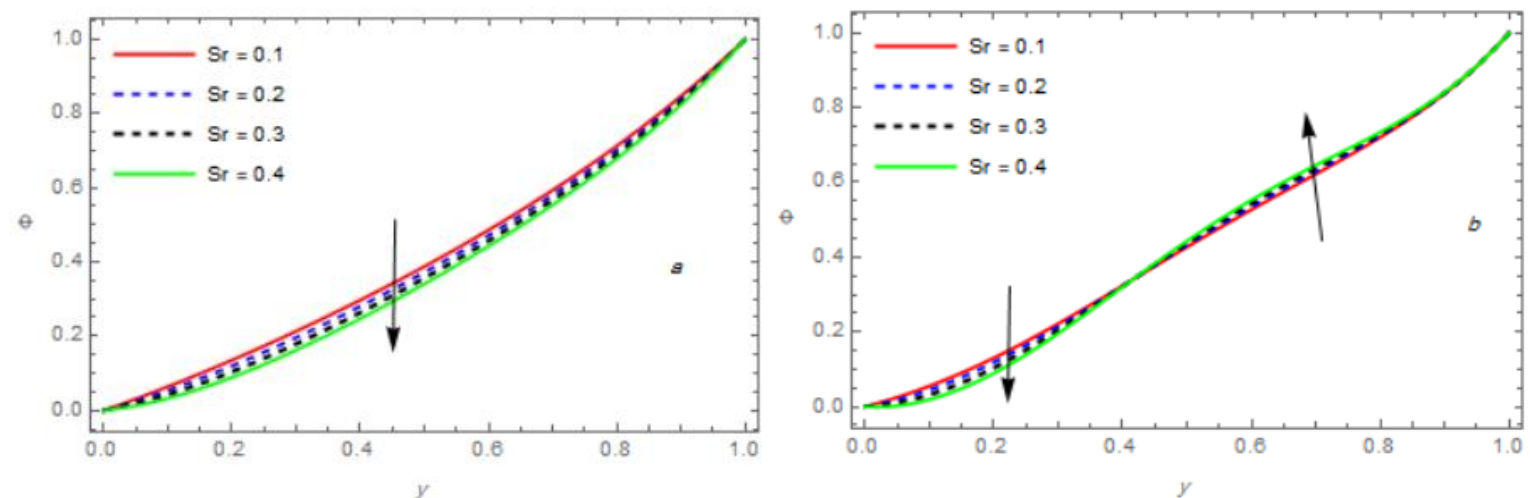

Fig 27 Influence of $S r$ on concentration for $B n=1, P e=2, \alpha=0.05, B r=1, \omega=1, M=$ $1, K_{r}=0.5, S c=0.6, t=0.5, D a=0.8, \lambda=1, R e=1, \xi=\frac{\pi}{4}, \delta=\frac{\pi}{4}, U_{0}=0.3, N=$ $1.25, \mathrm{Fr}=0.3$.
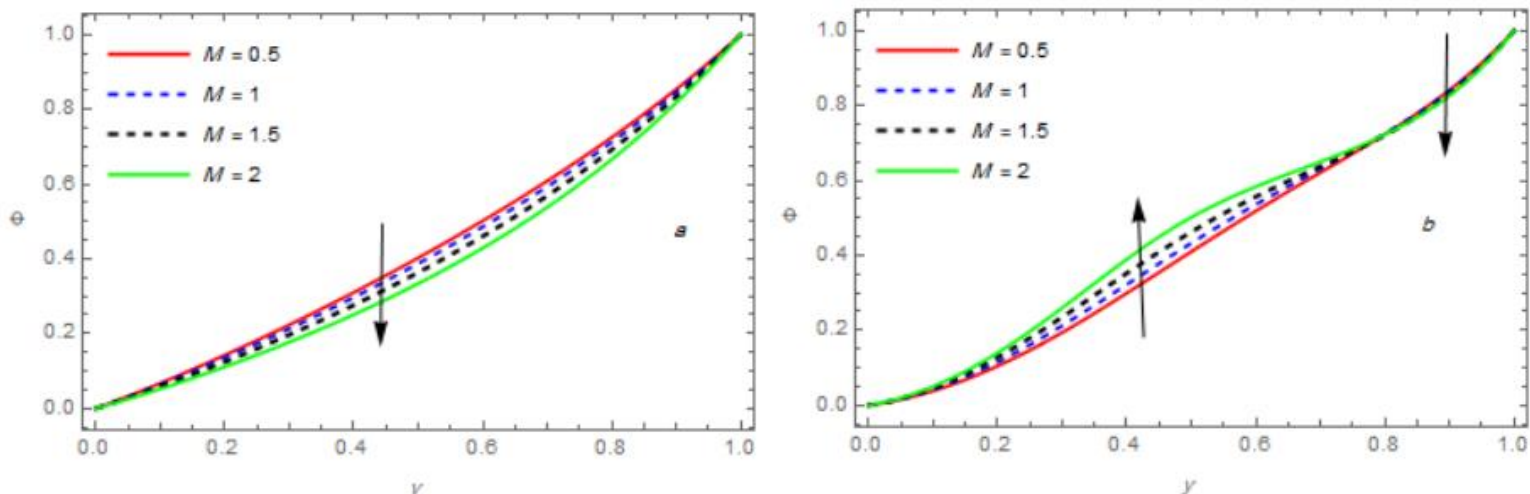

Fig 28 Influence of $M$ on concentration for $B n=1, P e=2, \alpha=0.05, B r=1, \omega=1, N=$ $1, K_{r}=0.5, S r=0.1, t=0.5, D a=0.8, \lambda=1, R e=1, \xi=\frac{\pi}{4}, \delta=\frac{\pi}{4}, U_{0}=0.3, S c=$ $0.6, \mathrm{Fr}=0.3$.
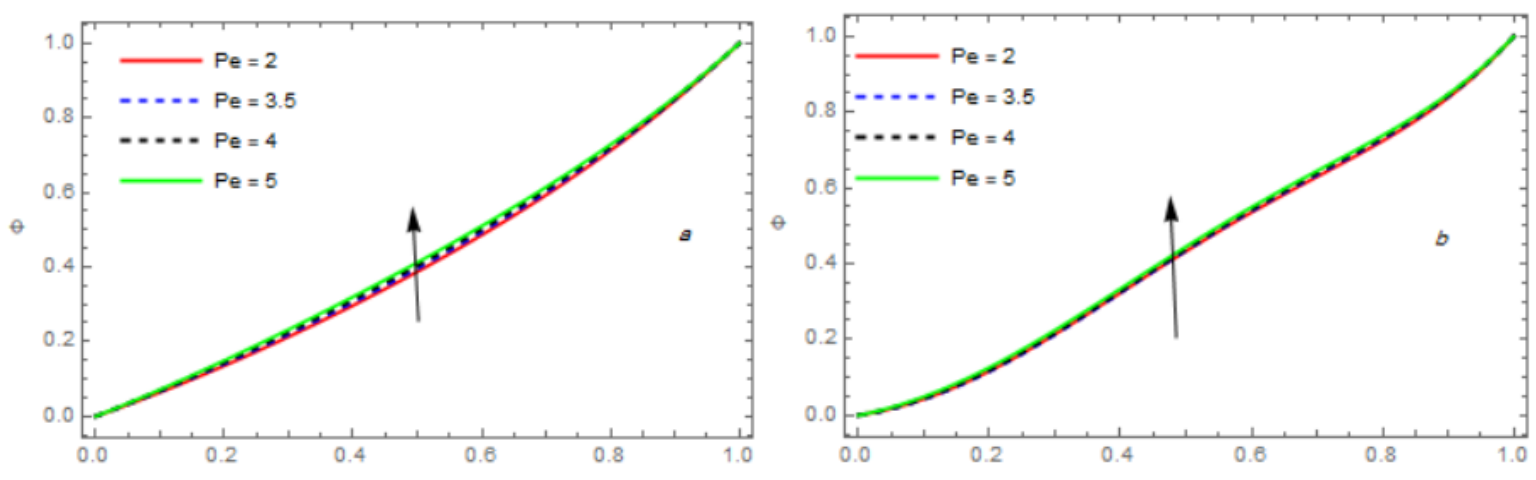

Fig 29 Influence of $P e$ on concentration for $B n=1, M=1, \alpha=0.05, B r=1, \omega=1, N=$ $1, K_{r}=0.5, S r=0.1, t=0.5, D a=0.8, \lambda=1, R e=1, \xi=\frac{\pi}{4}, \delta=\frac{\pi}{4}, U_{0}=0.3, S c=$ $0.6, \mathrm{Fr}=0.3$. 

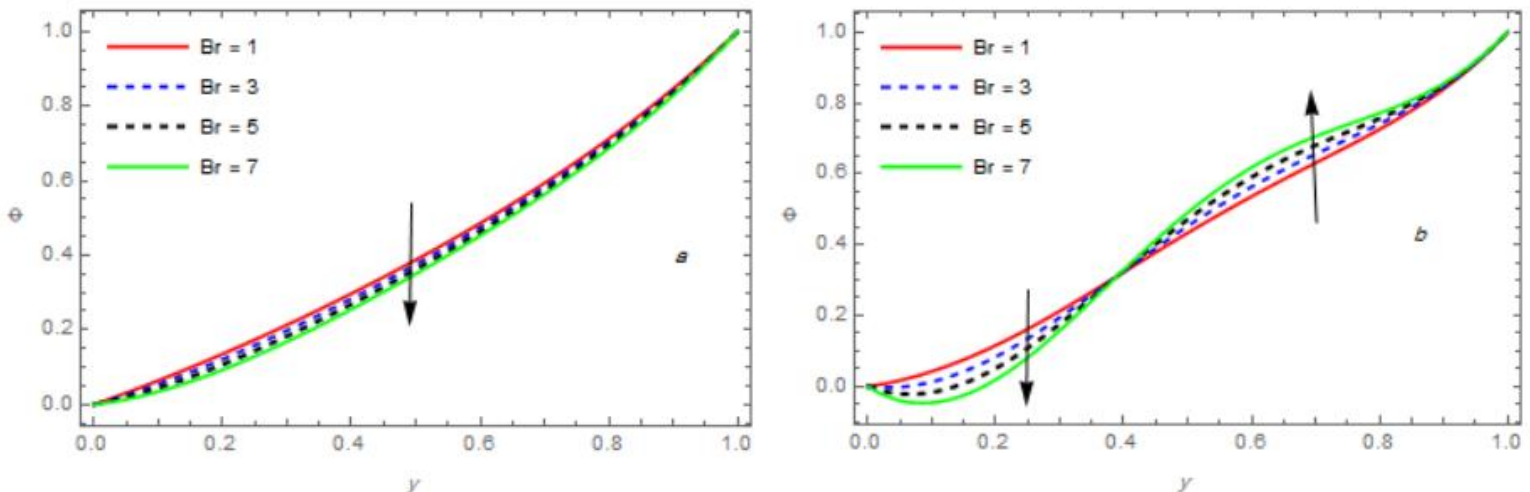

Fig 30 Influence of $B r$ on concentration for $B n=1, M=1, \alpha=0.05, P e=2, \omega=1, N=$ $1, K_{r}=0.5, S r=0.1, t=0.5, D a=0.8, \lambda=1, R e=1, \xi=\frac{\pi}{4}, \delta=\frac{\pi}{4}, U_{0}=0.3, S c=$ $0.6, F r=0.3$.
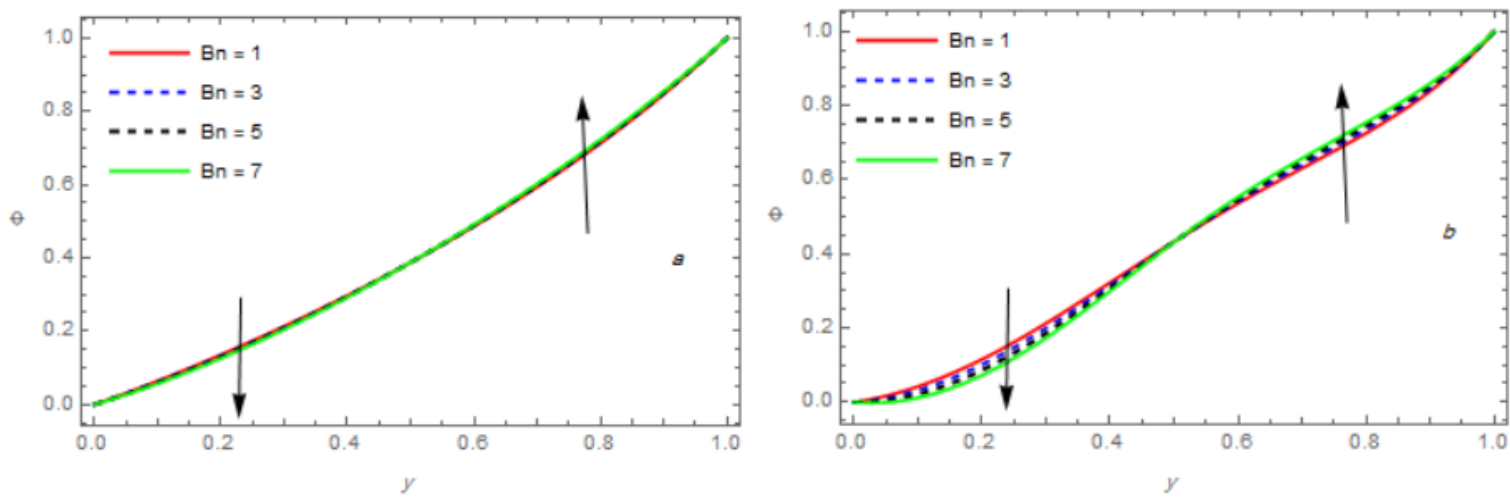

Fig 31 Influence of $B n$ on concentration for $B r=1, M=1, \alpha=0.05, P e=2, \omega=1, N=$ $1, K_{r}=0.5, S r=0.1, t=0.5, D a=0.8, \lambda=1, R e=1, \xi=\frac{\pi}{4}, \delta=\frac{\pi}{4}, U_{0}=0.3, S c=$ $0.6, F r=0.3$.
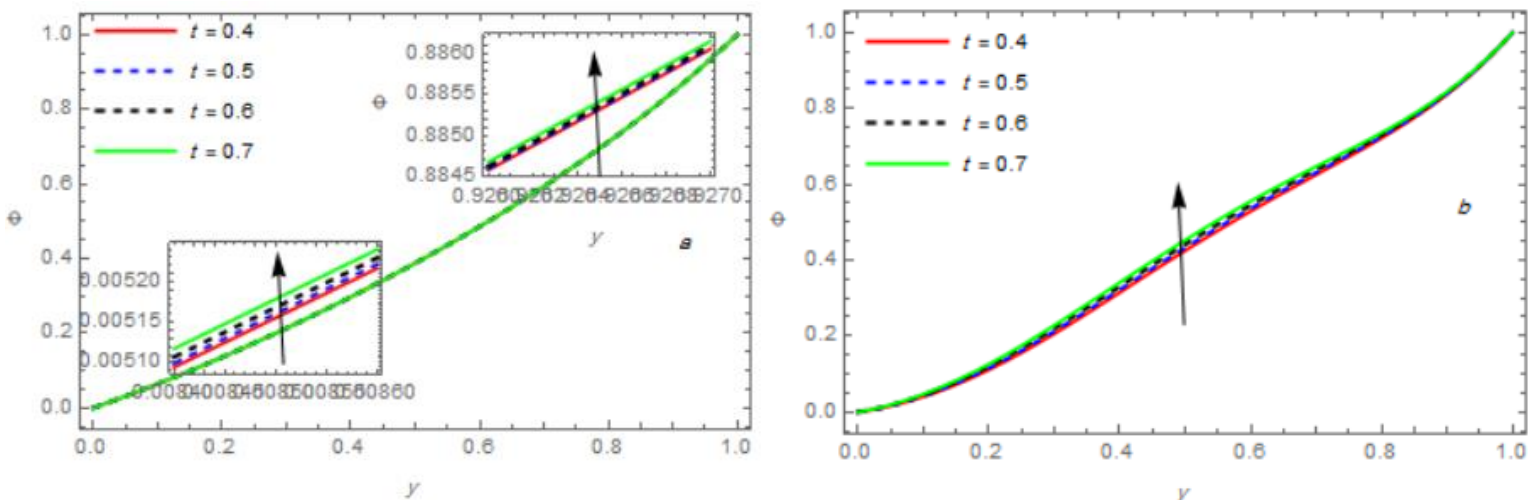

Fig 32 Influence of $t$ on concentration for $B n=1, M=1, \alpha=0.05, P e=2, \omega=1, N=$ $1, K_{r}=0.5, S r=0.1, B r=1, D a=0.8, \lambda=1, R e=1, \xi=\frac{\pi}{4}, \delta=\frac{\pi}{4}, U_{0}=0.3, S c=$ $0.6, F r=0.3$. 

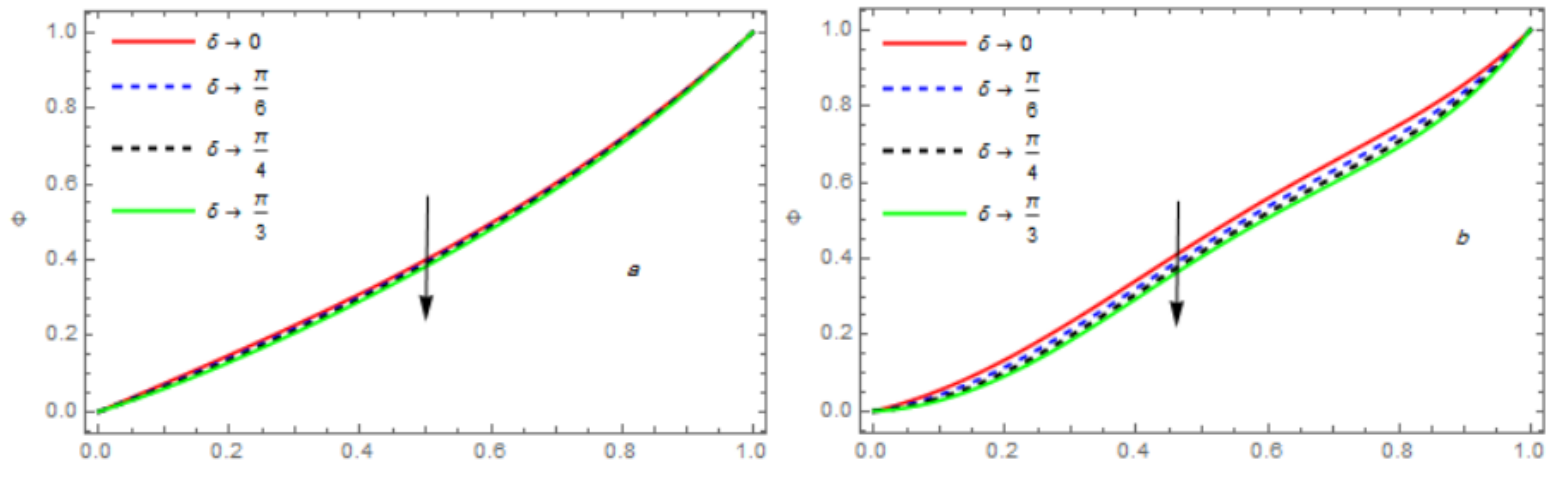

Fig 33 Influence of $\delta$ on concentration for $B n=1, M=1, B r=1, P e=2, \omega=1, N=$ $1, K_{r}=0.5, S r=0.1, t=0.5, D a=0.8, \lambda=1, \operatorname{Re}=1, \xi=\frac{\pi}{4}, U_{0}=0.3, S c=0.6, F r=0.3$.
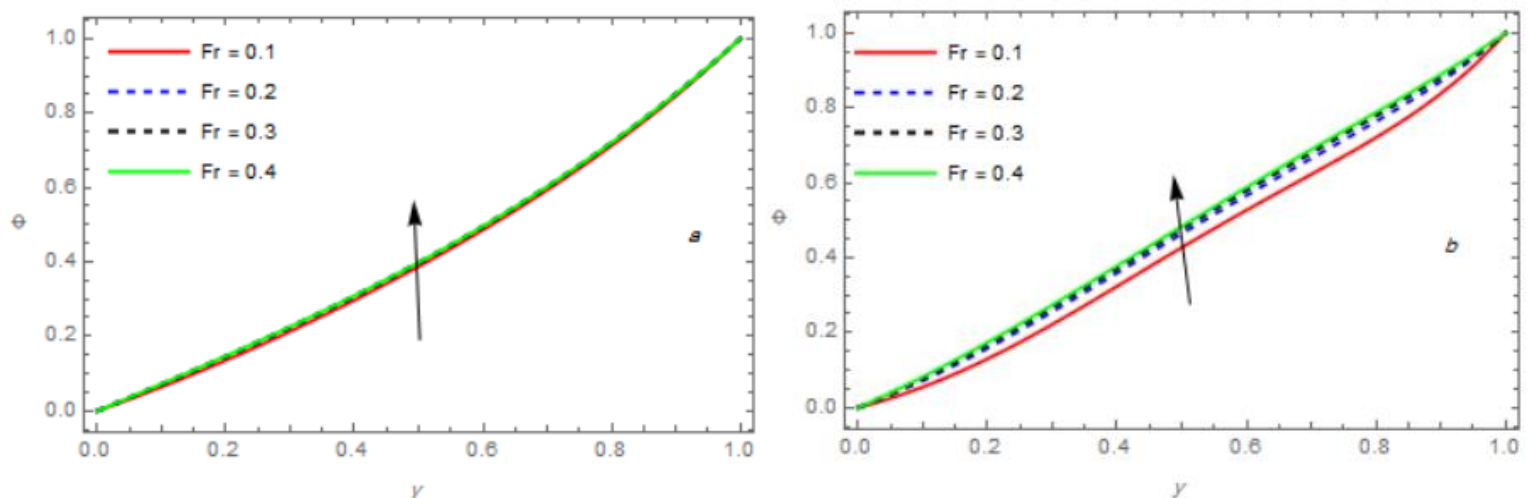

Fig 34 Influence of $F r$ on concentration for $B n=1, M=1, B r=1, P e=2, \omega=1, N=$ $1, K_{r}=0.5, S r=0.1, t=0.5, D a=0.8, \lambda=1, R e=1, \xi=\frac{\pi}{4}, \delta=\frac{\pi}{4}, U_{0}=0.3, S c=0.6$.
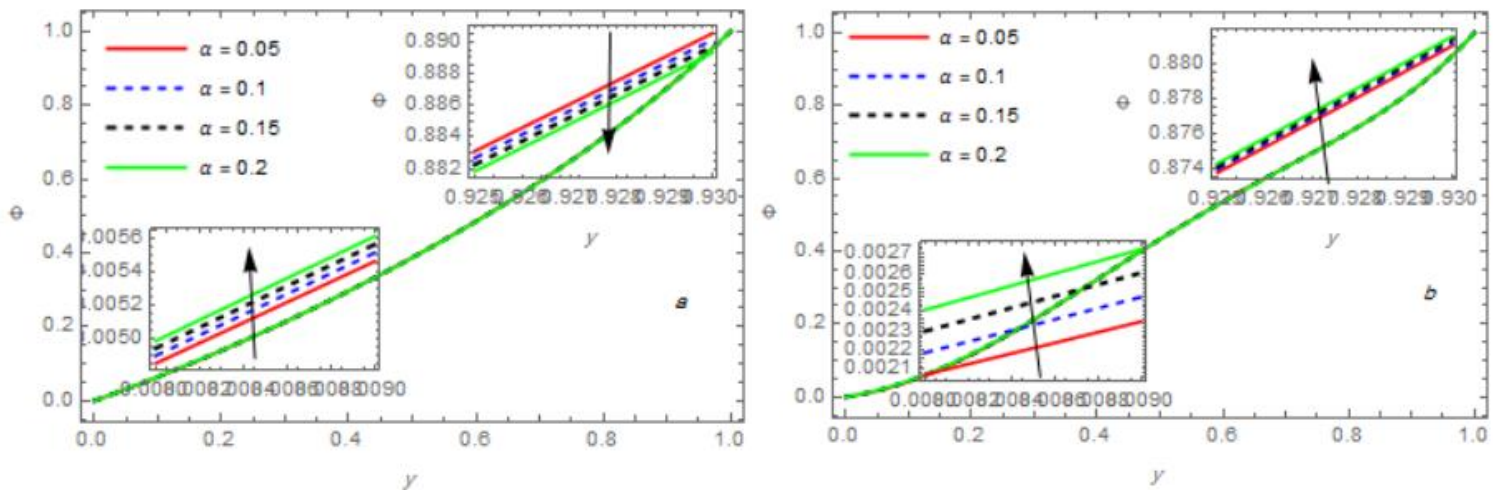

Fig 35 Influence of $\alpha$ on concentration for $B n=1, M=1, B r=1, P e=2, \omega=1, N=$ $1, K_{r}=0.5, S r=0.1, t=0.5, D a=0.8, \lambda=1, R e=1, \xi=\frac{\pi}{4}, \delta=\frac{\pi}{4}, U_{0}=0.3, S c=$ $0.6, \mathrm{Fr}=0.3$. 

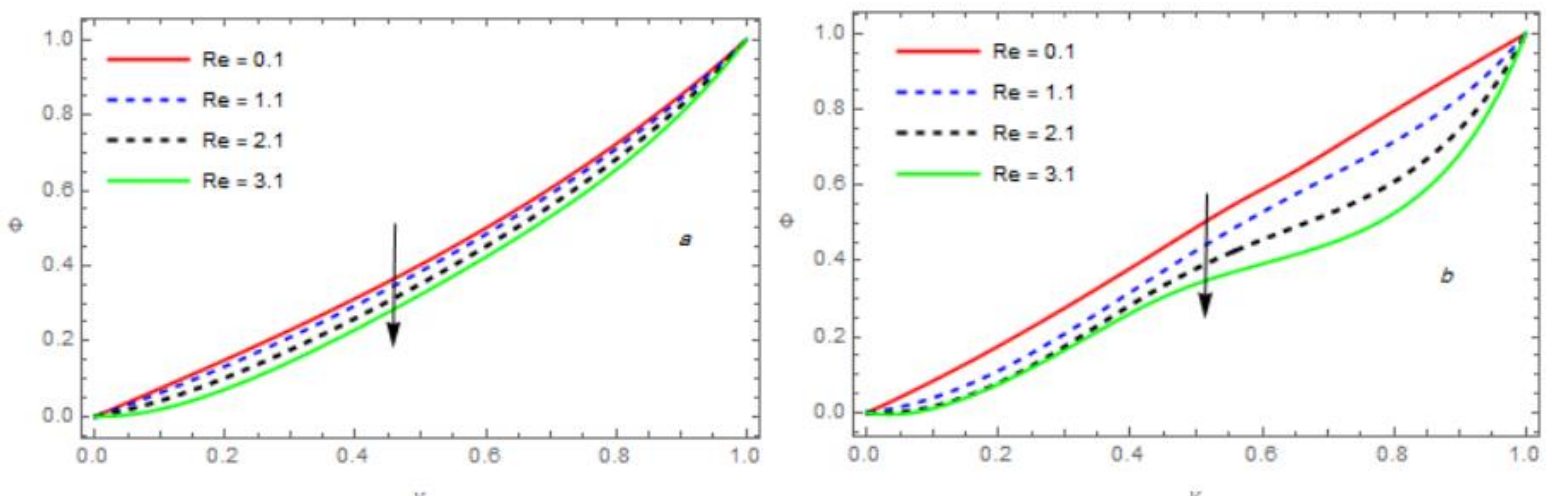

Fig 36 Influence of $R e$ on concentration for $B n=1, M=1, B r=1, P e=2, \omega=1, N=$ $1, K_{r}=0.5, S r=0.1, t=0.5, D a=0.8, \lambda=1, \alpha=0.05, \xi=\frac{\pi}{4}, \delta=\frac{\pi}{4}, U_{0}=0.3, S c=$ $0.6, F r=0.3$.
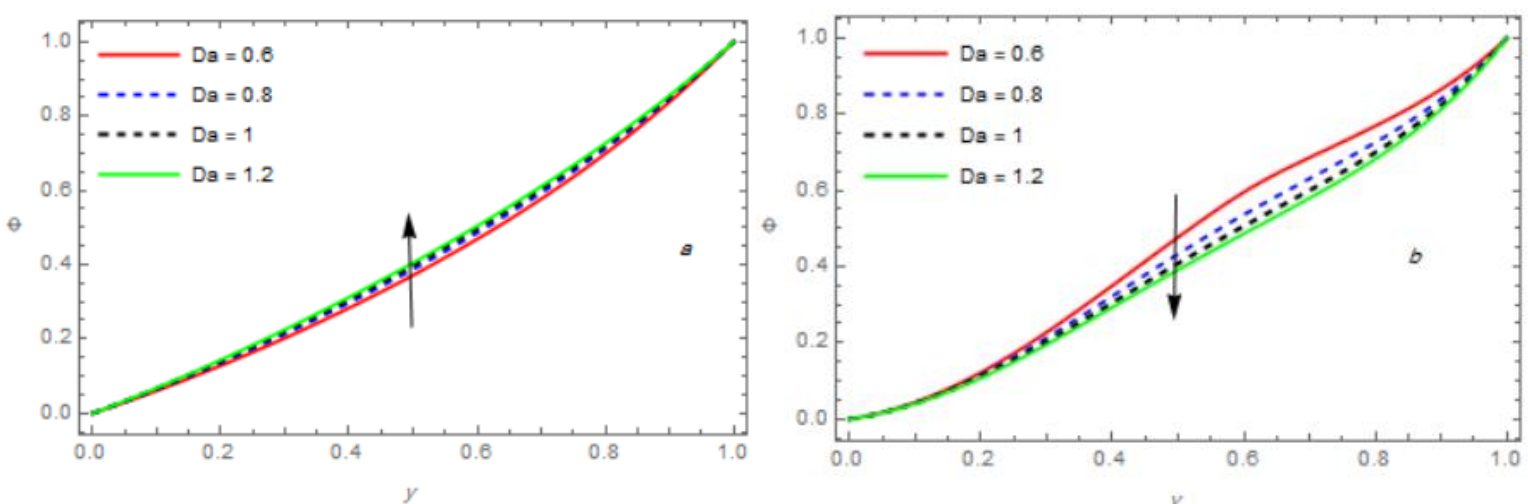

Fig 37 Influence of $D a$ on concentration for $B n=1, M=1, B r=1, P e=2, \omega=1, N=$ $1, K_{r}=0.5, S r=0.1, t=0.5, R e=1, \lambda=1, \alpha=0.05, \xi=\frac{\pi}{4}, \delta=\frac{\pi}{4}, U_{0}=0.3, S c=$ $0.6, \mathrm{Fr}=0.3$.
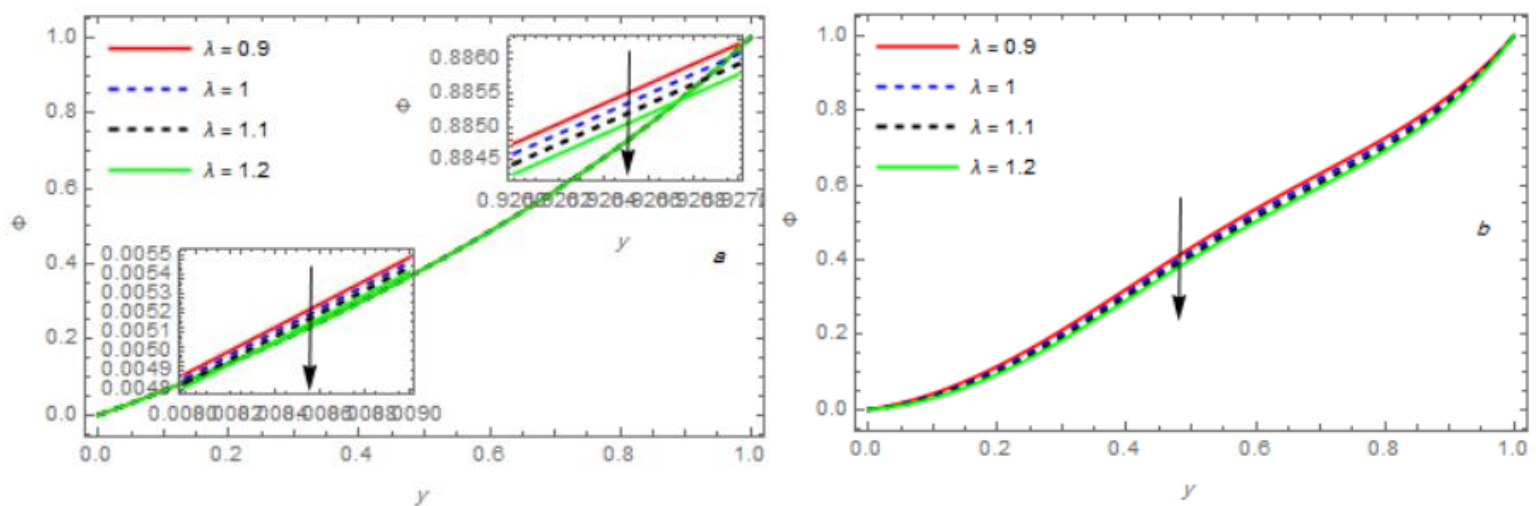

Fig 38 Influence of $\lambda$ on concentration for $B n=1, M=1, B r=1, P e=2, \omega=1, N=$ $1, K_{r}=0.5, S r=0.1, t=0.5, R e=1, D a=0.8, \alpha=0.05, \xi=\frac{\pi}{4}, \delta=\frac{\pi}{4}, U_{0}=0.3, S c=$ $0.6, \mathrm{Fr}=0.3$. 

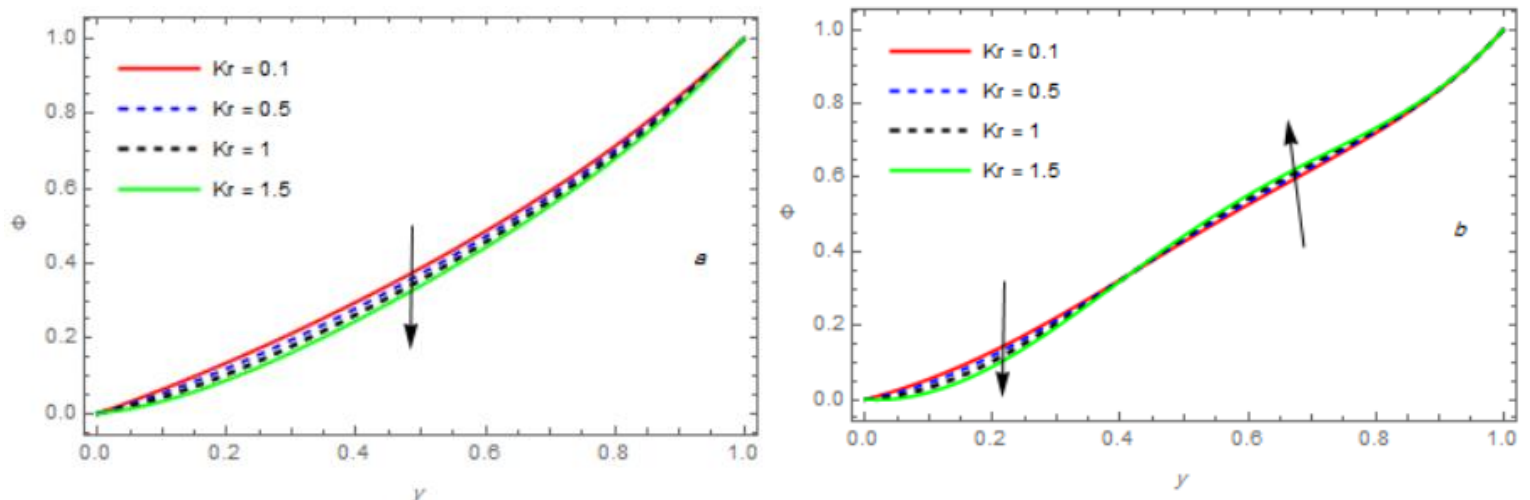

Fig 39 Influence of $K_{r}$ on concentration for $B n=1, M=1, B r=1, P e=2, \omega=1, N=$ $1, S r=0.1, t=0.5, R e=1, D a=0.8, \alpha=0.05, \xi=\frac{\pi}{4}, \delta=\frac{\pi}{4}, U_{0}=0.3, S c=0.6, F r=$ 0.3 .

\section{Conclusion}

This study, explaining the impacts of mass transfer on MHD oscillatory flow for Bingham fluid through a porous channel. We examined the influences of certain parameters that are active on fluid velocity by analyzing the graphs obtained after we reached the momentum equation solution, and used the MATHEMATICA program to plot the velocity and temperature and concentration of the fluid for two types of flow (Poiseuille and Couette).

- We used different values to find the results of pertinent parameters, namely Darcy number $(D a)$, Reynolds number $(R e)$, Peclet number $(P e)$, magnetic parameter $(M)$, Bingham number $(B n)$, Brinkman number $(B r)$, radiation parameter $(N)$.

- We show that the velocity profiles were increased by the increasing $D a, \lambda, \delta$ and $R e$ for both the Poiseuille and Couette flow.

- the velocity profiles were decreases by the increasing $t, M, F r$ and $\omega$ for both the Poiseuille and Couette flow.

- We show that the temperature profiles were decreases by the increasing $t, P e, M, F r$ and $\omega$ for both the Poiseuille and Couette flow.

- The temperature profiles were increased by the increasing $N, B r, D a, \operatorname{Re} \delta$ and $\lambda$ for both the Poiseuille and Couette flow.

- We show that the concentration profiles were increased by the increasing $P e, \omega, t$ and $F r$ for both the Poiseuille and Couette flow.

- the concentration profiles were decreases by the increasing $N, S c, R e, \delta, \lambda$ and $S r$, for both the Poiseuille and Couette flow.

\section{Reference}

[1] Y. J. Kim, Int. J. Eng. Sci., 38 , p. 833-845, (2000).

[2] Dheia G Salih Al -Khafajy, Lqaa Tareq Hadi. Influence of Heat Transfer on MHD Oscillatory Flow for Eyring-Powell Fluid through a Porous Medium with Varying Temperature and Concentration. Iraqi J. Sci., Vol. 61, No. 12, pp: 3355-3365. (2020). 
[3] Wissam Sadiq Khudair, Dheia G Salih Al-Khafajy, Influence of Heat Transfer on MHD Oscillatory Flow for Williamson Fluid with Variable Viscosity Through a Porous Medium, Int. J. Fluid Mechanics \& Thermal Sciences, 4(1): 11-17, (2018).

[4] Rasha Yousif Hassen Hayat A. Ali, Hall and Joule's heating Influences on Peristaltic Transport of Bingham plastic Fluid with Variable Viscosity in an Inclined Tapered Asymmetric Channel, Ibn Al-Haitham J. Pure \& Appl. Sci. 34 (1), (2021).

[5] Al-Khafajy, D.G., Effects of Heat Transfer on MHD Oscillatory Flow of Jeffrey Fluid with Variable Viscosity through Porous Medium. Advances in Applied Science Research,7(3),pp.179186, (2016).

[6] Farah Alaa Adnan; Ahmad, M. Abdulhadi. Effect of an inclined magnetic field on peristaltic flow of Bingham plastic fluid in an inclined symmetric channel with slip conditions, Iraqi J. of Science. 60, 7, 1551-1574, (2019).

[7] Farah A. Adnan and Ahmad M. Abdul Hadi, Effect of an inclined magnetic field on peristaltic flow of Bingham plastic fluid in an inclined symmetric channel with slip conditions, Iraqi Journal of Science, 60(7), 1551-1574, (2019).

[8] Sleigh A; An introduction to fluid mechanics, Lecture course, cive 1400 fluid mechanics, (2001).

[9] Soundalgekar VM and Bhat J., Oscillatory MHD channel flow and heat transfer. Indian J. Pure Appl. Mam. 15(7), 819-28, (1984).

[10] Bénard H., Les Tourbillons cellulaires dans une nappe liquide,Revue générale des Sciences pures et appliquées, (11), pp.1261-1271, (1900).

[11] Rasha Yousif Hassen Hayat A. Ali, Hall and Joule's heating Influences on Peristaltic Transport of Bingham plastic Fluid with Variable Viscosity in an Inclined Tapered Asymmetric Channel, Ibn Al-Haitham J. Pure \& Appl. Sci. 34 (1), (2021). 